\title{
EchoGéo
}

$58 \mid 2021$

Varia

\section{Faire pays dans un cyberespace : le cas des Haïtiens en Île-de-France}

Rébecca Cadeau

\section{OpenEdition}

1 Journals

Édition électronique

URL : https://journals.openedition.org/echogeo/22749

DOI : 10.4000/echogeo.22749

ISSN : 1963-1197

Éditeur

Pôle de recherche pour l'organisation et la diffusion de l'information géographique (CNRS UMR 8586)

Référence électronique

Rébecca Cadeau, «Faire pays dans un cyberespace : le cas des Haïtiens en Île-de-France », EchoGéo [En ligne], 58 | 2021, mis en ligne le 31 décembre 2021, consulté le 12 février 2022. URL : http:// journals.openedition.org/echogeo/22749; DOI : https://doi.org/10.4000/echogeo.22749

Ce document a été généré automatiquement le 12 février 2022.

EchoGéo est mis à disposition selon les termes de la licence Creative Commons Attribution - Pas d'Utilisation Commerciale - Pas de Modification 4.0 International (CC BY-NC-ND) 


\title{
Faire pays dans un cyberespace : le cas des Haïtiens en Île-de-France
}

\author{
Rébecca Cadeau
}

\section{Introduction}

1 Si le séisme du 12 janvier 2010, ayant causé environ 300000 morts, a mis Haïti à la une des médias internationaux, depuis, les mouvements sociaux, les manifestations contre la corruption et l'autoritarisme du Parti haïtien Tèt kale ${ }^{1}$ (PHTK) et les appels à la solidarité internationale ${ }^{2}$ (de 2018 à 2021) peinent à être visibles. Or, dans la communauté haïtienne ${ }^{3}$ de l'étranger dont le nombre s'élèverait à environ 1,8 million d'individus (Bidegain, 2013), des acteurs associatifs, qui se mobilisent lors des catastrophes et des crises, s'expriment. En France, cette communauté, considérée comme une diaspora, au regard, entre autres, des liens entretenus avec le pays d'origine et de l'organisation d'échanges entre les pôles (Audebert, 2011), œuvre à « une mise en visibilité dans l'espace public » (Cossée, 2010) afin de rendre la question haïtienne plus manifeste. Ces actions sont également révélatrices de connexion entre les divers lieux d'établissement des Haïtiens, mettant en évidence une concentration d'acteurs.

2 Alors que ce groupe représente, selon l'INSEE, une part importante des immigrés sur le plan quantitatif, seulement quelques études lui ont été consacrées en France dont une grande part concerne la Guyane. Pourtant, il compte officiellement 90000 individus en 2020 dont près de 37000 en île-de-France, d'où le choix de faire nos recherches dans cette région. Il constitue la plus importante communauté étrangère en provenance des Caraïbes et est de plus en plus structuré (presque 300 associations) ${ }^{4}$. André Calmont et Cédric Audebert (2003), en étudiant les dynamiques migratoires dans le Bassin caraïbe, constataient que $10 \%$ des 130 millions d'habitants vivaient à l'extérieur de leur pays d'origine; ce qui en fait un espace majeur des migrations internationales depuis les années 1950. Les dynamiques migratoires haïtiennes participent fortement à celles de la région et, à une échelle plus large, aux migrations internationales. La part résidant à 
l'étranger représenterait $20 \%$ de la population haïtienne selon Cédric Audebert (2012). Plus de $11 \%$ a émigré vers les États-Unis, la République dominicaine, le Canada, la France hexagonale, les Bahamas et les départements français d'Amérique (principalement la Guadeloupe et la Guyane) ${ }^{5}$, représentant les pôles majeurs, en 2015. Si les trois premières destinations s'expliquent par la proximité géographique (comme c'est aussi le cas pour les Bahamas), et les politiques de recrutement de main d'œuvre dans la Caraïbe, des liens historiques et culturels rapprochant les sociétés haïtienne et française depuis l'esclavage et la colonisation (XVII ${ }^{e}$ XIX ${ }^{e}$ siècles) expliquent le « choix » des territoires français.

3 Dans le champ des sciences humaines et sociales en France, des chercheurs comme Roger Bastide, Françoise Morin et François Raveau (1974) ont fait de cette migration leur objet d'étude. Claude Delachet-Guillon (1996), Margarita Mooney, (2008), Maud Laëthier (2011), Dimitri Béchacq (2010), James Lamare (2019) et Rodelin Exavier (2020) ont étudié les associations haïtiennes. Les travaux récents témoignent à la fois de l'affirmation du phénomène et de son intérêt grandissant pour les chercheurs.

4 Dans la géographie contemporaine française et francophone, Catherine Georgon (1985) étudie le caractère clandestin de l'immigration haïtienne en Guyane ainsi qu'un processus de bidonvilisation de la périphérie de Cayenne. De concert avec Régine Calmont, Catherine Georgon (1987) analyse les mouvements migratoires d'Haïti vers la Guyane alors que Régine Calmont, dans une thèse de doctorat sur «les migrations et les migrants en Guyane ", s'est concentrée sur la communauté haïtienne (1988). André Calmont $(1993,2007)$ s'est intéressé tantôt à l'intégration des Haïtiens, tantôt aux trajets socio-identitaires des jeunes issus de l'immigration haïtienne. De leur côté, Frédéric Piantoni et Zéphirin Romanovski (2008) ont décrit les stratégies d'accès au logement des Haïtiens dans l'agglomération de Cayenne. Cédric Audebert (2012) analyse les dynamiques sociales et spatiales construisant l'espace migratoire haïtien. Quant à Clara Rachel Eybalin Casséus (2013), elle étudie les stratégies des Haïtiens en France et leur impact socioéconomique et politique sur la société d'origine, selon une approche comparative avec la communauté jamaïcaine vivant en Grande Bretagne. Sur le continent américain, on peut également citer les travaux de Violaine Jolivet sur les territorialités des Haïtiens (2017) ; ceux de Sophie Lanno-Cyr (2016) sur les mobilités de cette communauté, ses pratiques transnationales et les incidences sur les concepts de territoire et d'identité; le livre de Zéphirin Romanovski (2016) sur les réseaux migratoires haïtiano-guyanais; l'article de Pascale Smorag (2019) sur les espaces haïtiens de Miami comme Little Haïti ou les nouveaux quartiers du Nord de la ville et le livre de Regine O. Jackson (2011) dans lequel l'étude spatiale de la diaspora haïtienne se décline en plusieurs géographies.

5 En Haïti, depuis les recherches de Georges Anglade $(1974,1982,1990)$ dont une partie est consacrée aux mouvements de population et à l'évolution démographique, peu de travaux empiriques portent sur les phénomènes migratoires. Ceux de Georges Eddy Lucien $(2009,2015)$ sur les transformations territoriales de Verrettes (Département de l'Artibonite) et la gentrification de Little Haïti à Miami et ceux de JeanClaude Icart (1987) dont un article (2017) faisant un bilan de l'évolution des migrations haïtiennes de 1970 à nos jours, sont les seuls à notre connaissance. Tandis que les associations haïtiennes rendent visibles, dans des lieux donnés, des moments forts de sociabilité et d'apprentissage politique, rares sont les recherches de géographie française, francophone sur Haïti et de géographie haïtienne qui privilégient une entrée 
par ces structures. Pourtant, elles s'avèrent un objet intéressant pour l'analyse des processus spatiaux, conférant aux diasporas la capacité de rapprocher les lieux d'origine et d'installation.

6 Parmi les travaux majeurs sur les territorialités des diasporas, ceux d'Emmanuel Ma Mung $(1994,2012)$ sur la diaspora chinoise et d'Anthony GoreauPonceaud (2008) sur la diaspora tamoule retiennent notre attention. Leur analyse de la territorialité des populations étudiées forme un cadre propice pour appréhender les dynamiques mises en œuvre par les associations haïtiennes en Île-de-France témoignant d'une conscience de leur dispersion par les relations entreprises avec les autres pôles, leur représentation dans l'espace et les marqueurs esquissant une "communauté imaginée» (Anderson, 2006). En ce sens, les recherches de Cédric Audebert (2012) ont confirmé la création de réseaux familiaux et régionaux haïtiens et de stratégies transnationales permettant de circuler dans l'espace « réel » de cette diaspora. Notre propos s'appuiera sur les travaux de ces auteurs bien qu'il convienne ici de considérer l'espace, au sens physico-spatial aussi bien qu'au sens virtuel, servant de support à la construction des réseaux diasporiques (Berthomière et Hassane, 2009; Diminescu, 2005; Marchandise, 2010) depuis le développement des Technologies d'Information et de Communication (TIC). Dès lors, l'analyse de ces dernières paraît indispensable à "l'étude géographique d'une population en diaspora » (Marchandise, 2010).

7 En s'intéressant à l'espace numérique des associations haïtiennes, notre travail vise à contribuer à l'avancement des réflexions sur la structuration spatiale des migrants via les TICs. Il s'agit de comprendre l'impact des outils technologiques utilisés par des Haïtiens déterritorialisés dans un processus de reterritorialisation à l'échelle d'un groupe. Les considérations se limitent au cadre des dynamiques de la branche non religieuse $^{6}$ du secteur associatif haïtien en Île-de-France. Elles tenteront d'articuler des espaces dits réel et virtuel de la diaspora haïtienne à partir d'entretiens et d'informations recueillies sur le web. Quelles sont les pratiques socio-spatiales en usage dans cette communauté dont la visibilité semble être de plus en plus manifeste, notamment dans les actions visant à l'émancipation de la société d'origine? Quels sont les impacts spatiaux induits par ces pratiques? Le phénomène de concentration d'acteurs, pouvant rendre la présence haïtienne plus visible dans l'espace public, est-il propre à cette communauté ? Afin de répondre à ces interrogations, le propos est organisé en quatre parties : la première présente les principales vagues de la migration haïtienne; la deuxième décrit la structuration spatiale de la communauté en île-deFrance, la troisième et la quatrième analysent les territorialités des acteurs associatifs. Si l'étude de la territorialité des acteurs, tant dans l'espace réel que virtuel, et de la configuration des liens à travers des réseaux socionumériques ${ }^{7}$ aide à comprendre les rôles et l'importance des événements communautaires, nous souhaiterions ici mettre l'accent sur le rapprochement des territoires vécus par un groupe dispersé et les types d'actions lui permettant de construire un espace transnational.

\section{Méthodes et données}

8 Les données mobilisées dans cet article proviennent d'une enquête qualitative dans le cadre de ma thèse de doctorat de géographie, en cours, sur la territorialisation des Haïtiens en Île-de-France. Les résultats constituent les fruits de la phase exploratoire, 
de septembre 2019 à décembre 2020, durant laquelle j'ai dénombré 40 associations haïtiennes et franco-haïtiennes et participé à une trentaine d'événements ainsi qu'à une vingtaine de réunions organisées en Île-de-France. Les périodes propices aux observations sont celles-ci : rentrée universitaire, salon du livre haïtien (mois de novembre ou décembre), fêtes nationales haïtiennes (voir l'illustration 3), fête des morts ( $1^{\text {er }}$ et 2 novembre), fêtes de fin d'année, saison des bals. Mais également des festivals, des soirées de gala, etc. Autant de moments où cette communauté regroupe individus, familles et réseaux d'interconnaissance. La newsletter hebdomadaire du service culturel de l'Ambassade d'Haïti en France fait état d'une vingtaine d'événements par mois en moyenne, soit autant de rassemblements susceptibles de permettre des enquêtes sur le fonctionnement d'un espace migratoire haïtien.

Par ailleurs, j'ai observé l'espace numérique de ces acteurs associatifs (Facebook, Whatsapp, Zoom), surtout pendant les périodes de restriction à l'espace public marquant la crise épidémiologique de 2020 et 2021. Sur les pages, les forums et les groupes des associations, ont été pris en compte les post ayant un lien avec le pays d'origine. Dans le corpus constitué, ces post sont majoritaires et se présentent sous diverses formes: vidéos, photographies, affiches, tracts, textes écrits (articles, notes de presse, lettres, et parfois des tirades) messages vocaux, sujets de débats, etc. Les données de l'article puisent leur source dans l'espace numérique de six acteurs parmi les plus actifs que nous enquêtons : les sites de l'Association pour le Rayonnement culturel d'Haïti et de son Environnement (ARCHE) et Espace des Haïtiens Vivant à l'Etranger (Espace-HVIE), les pages Facebook de Haïti Plus juste (HPJ), de ARCHE et du Mouvement pour une Candidature unique (MCU) ainsi que les forums whatsapp du Conseil représentatif de la Communauté haïtienne de France (CORECOHF), du Mouvement haïtien de France (MHdF) et de MCU. Les post, les messages et les images des réseaux sociaux et des sites que nous en avons extraits feront l'objet de nos analyses.

\section{Les principales vagues de la dispersion des Haïtiens}

La dynamique migratoire haïtienne est à la fois ancienne et massive proportionnellement à la population. Elle concerne les deux côtés de l'Atlantique (illustration 1) et se structure en plusieurs vagues. 
Illustration 1 - Distribution de la population à l'échelle mondiale

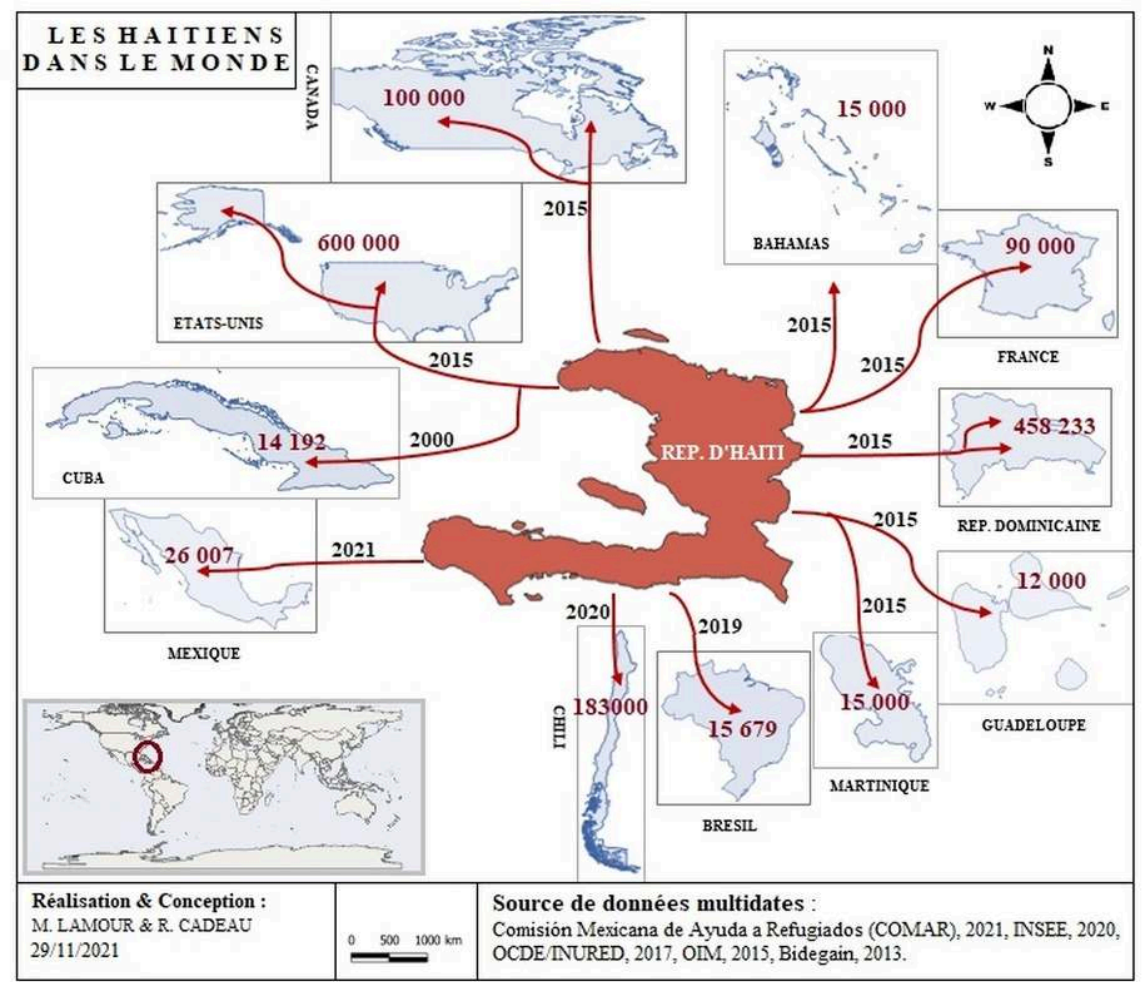

Cette carte est inspirée de celle de Georges Anglade (2005) sur les flux migratoires haïtiens au $X X X^{e}$ siècle. Elle représente les principales destinations des flux d'Haïti vers les pôles majeurs de cette migration. L'Amérique du nord constitue le principal bassin d'attraction.

11 Haïti est un pays de $27700 \mathrm{~km}^{2}$ qui forme, avec la République dominicaine, l'une des quatre îles des Grandes Antilles, baignées par la mer des Caraïbes. Sa population actuelle est d'environ 11 millions d'habitants selon la Banque mondiale.

Ancienne colonie espagnole et française fondée sur l'exploitation des mines (principalement de l'or) suivie de l'agriculture d'exportation (le sucre, le coton, l'indigo, le café, le cacao) après quelques siècles (Lucien, 2018 ; Madiou, 1989), plus de 1 200 vaisseaux accostaient dans les ports de ce territoire dont les productions de sucre et de café atteignent $40 \%$ et $60 \%$ de la production mondiale (Lucien, 2018, p. 80). Après l'ultime bataille contre l'armée française, le 18 novembre 1803 à Vertières (ville du département $\mathrm{du}$ Nord), naît Haïti le $1^{\mathrm{er}}$ janvier 1804 . Après l'indépendance, les exportations haïtiennes perdirent progressivement leur place sur le marché mondial et, à partir de la fin du XIXe, la paysannerie haïtienne, de plus en plus nombreuse et appauvrie, a connu des mouvements d'émigration.

Une première vague s'est produite à partir de 1900 (Audebert et Calmont, 2003), où environ $20 \%$ de la population partit en direction de la République dominicaine et de Cuba (Lucien, 2015). Ces flux augmentent considérablement à partir de 1913 (Domenach, 1985) pour exploser avec l'Occupation états-unienne en Haïti entre 1915-1934 dans le contexte de l'expansionnisme étasunien dans la région. Ils auraient été multipliés par 20 entre 1914 et 1915 (Audebert, 2012) en même temps que la mise en place d'une migration d'études vers l'Amérique du Nord et l'Europe se dessine (Lucien, 2015). Malgré l'apparition de nouvelles destinations, Cuba et la République dominicaine 
demeurent, avec d'autres îles de la région, les premiers pôles de la migration internationale haïtienne.

Le déclin de l'activité sucrière dans les pays caribéens, causé, entre autres, par une chute globale du prix du sucre ${ }^{8}$ et la crise économique de 1929 ont inversé les flux vers Cuba et la République dominicaine dont les gouvernements ne se montrent plus accueillants envers les migrants étrangers. Une ambiance de répression s'installe dans ces lieux d'installation et l'année 1937 marque le massacre de 20000 à 30000 Haïtiens dans la république voisine à l'instigation du dictateur Rafael Léonidas Trujillo (Anglade, 1982 ; Hurbon, 1987). Les industriels et les investisseurs ferment l'activité sucrière dans la région, les émigrés haïtiens qui rentrent au bercail contribuent à l'augmentation du chômage dans le pays. Cette situation touche plusieurs couches sociales et engendre deux vagues migratoires : l'une interne, avec des flux importants vers la capitale Portau-Prince et l'autre internationale, en direction de l'Amérique du Nord. Ce moment historique semble fondamental à «la constitution des premiers réseaux migratoires internationaux de ce pays » (Cédric Audebert, 2012, p. 22). La présence haïtienne s'y est manifestée dans les années 1940, notamment par la création de la première association haïtienne à l'étranger (New-York) par des étudiants : l'Union démocratique haïtienne (Lucien, 2015).

Une vague, plus hétérogène sur les plans sociologique et géographique, fut provoquée par la dictature des Duvalier (1957-1986) et le marasme économique de la période. Le premier groupe était composé des membres de l'élite mulâtre ainsi que d'opposants au régime. Les intellectuels et les classes moyennes noires les suivirent, après l'autoproclamation de François Duvalier comme président à vie en 1964 (Audebert, 2012 ; Béchacq, 2007, 2010 ; Saint Victor, 2018). Des professionnels de la classe moyenne (médecins, enseignants, techniciens, etc.) répondent aux appels d'offre de l'Organisation des États américains (OEA), d'autres organisations internationales et des nouvelles nations africaines pour aller travailler en Afrique (Lucien, 2015). À la fin de leur contrat, en 1968, une bonne portion a migré vers le Canada, pour pallier le manque de cadres, et vers les États-Unis (Jadotte, 1977). L'immigration états-unienne compta 40 100 immigrants et 100000 non immigrants venant d'Haïti en 1971 (Audebert, 2012). Des raisons politiques autant d'ordre interne qu'externe poussent des cadres, des professionnels libéraux et des employés qualifiés à continuer à quitter le pays.

Selon Paul Dejean (1990), la migration haïtienne est plus massive, définitive et dispersée à partir de 1968 : les pôles se diversifient (Montréal, Miami, Petites Antilles, etc.) ; les effectifs s'intensifient. Les côtes de la Floride, de par leur proximité avec la Caraïbe, deviennent une entrée idéale pour l'émigration illégale pratiquée par des boat people ${ }^{9}$ haïtiens et cubains (Audebert, 2003 ; Audebert et Calmont, 2007). De 1981 à 1997, 50000 à 70000 boat people haïtiens débarquèrent sur les côtes de la Floride (Dejean, 1990 ; Jolivet, 2015). En même temps que ces flux dont une part est refoulée au territoire d'origine, d'autres se profilent vers la France, la Belgique ou l'Espagne où le visa d'entrée n'était pas encore obligatoire (Delachet-Guillon, 1996). D'un autre côté, les migrations intra-caribéennes s'amplifient. Cédric Audebert (2007) remarque que les départements français d'Amérique ont accueilli, au cours du $\mathrm{XX}^{\mathrm{e}}$ siècle, pléthore de migrants en provenance des territoires voisins. La Guyane française, ayant connu plusieurs contextes favorisant l'immigration comme la colonisation au XIX ${ }^{\mathrm{e}}$ siècle, la départementalisation en 1946 et la construction du centre de Kourou entre 1965 et 1968 
(Calmont, 1993; Georgon, 1985; Piantoni, 2016; Laëthier, 2011), est un terrain privilégié de la migration haïtienne ${ }^{10}$.

17 En France métropolitaine, la création du BUMIDOM (Bureau pour le développement des Migrations dans les Départements d'Outre-Mer) en 1963 a encouragé des milliers d'Antillais à migrer vers l'ancienne métropole (Milia-Marie-Luce, 2007) dans un contexte de mutations socio-économiques dans les anciennes colonies françaises d'Amérique et de besoin de main d'œuvre dans l'ancienne métropole provoquant des mouvements des Antilles vers la France hexagonale et de l'Europe vers les Antilles (Girault, Dubost, Calmont et al., 2009; Haddad, 2018). Certains de nos enquêtés connaissent des compatriotes ayant émigré d'Haïti vers la Guyane française puis de là en métropole. Vers l'île-de-France, deux grandes vagues semblent se produire dans les années 1970 et 1980. La première se manifeste par une forte mobilité des élites haïtiennes. Margarita Mooney (2008) relate d'ailleurs que beaucoup d'Haïtiens de cette vague ont fondé des associations, après la loi du 9 octobre 1981 (octroyant à nouveau aux étrangers le droit de créer des associations). La deuxième vague représente une émigration massive d'origine populaire provoquée par les crises politiques et la dégradation de l'économique (Louis-Jacques, 2015). Elle est formée majoritairement de femmes destinées à travailler comme femmes de ménage ou employées de maison chez des familles françaises. Cette vague s'amplifie avec l'arrivée de familles provenant du Sud d'Haïti ayant transité par la Guyane (Delachet-Guillon, 1996). Elle aurait vu naître de nombreuses associations et quelques émigrés originaires de la partie nord de l'île (Artibonite et Cap-Haïtien), ayant résidé dans la capitale, Port-au-Prince.

Dans un contexte de terreur post-duvaliériste, de ripostes progressiste et populaire et d'embargo par l'OEA, Haïti a aussi connu des vagues migratoires pendant les deux mandats du président Jean-Bertrand Aristide (1990-1995 et 2000-2005). En 1991, après le coup d'État organisé par les Forces armées d'Haïti, et une tentative d'assassinat du président élu (en septembre 1990), une forte émigration s'est dirigée principalement vers les États-Unis (Audebert, 2012). Claude Delachet-Guillon (1996) souligne que des femmes ayant participé aux campagnes d'alphabétisation du président exilé ont émigré vers la France, sans en donner le chiffre. Une nouvelle vague peut être identifiée en 2004. Devant l'augmentation annuelle de l'inflation, la baisse réelle du salaire minimum et la dévaluation continue de la gourde (monnaie d'Haïti), une crise politique pousse le président Aristide (qui était revenu en 1994) ainsi qu'une part de la population à l'extérieur du pays. Cédric Audebert (2012) considère l'émigration contemporaine non seulement comme une réponse ponctuelle et provisoire à l'État duvaliériste mais aussi une donnée structurelle d'après la dictature parce que, depuis, un sentiment d'insécurité plane en Haïti. Ajoutons que le séisme de 2010 et les crises qui s'en suivent ont engendré de nouvelles vagues aussi bien vers les anciennes destinations (Jolivet, 2017) que vers de nouvelles: Équateur, Brésil, Chili, Argentine (Louidor, 2011) et Mexique. On assiste à leur remontée spectaculaire vers ce dernier pays ${ }^{11}$ servant d'espace transit dans la perspective de fouler le sol étasunien en bravant le mur de Donald Trump.

19 Comment une dynamique migratoire aussi intense, ancienne et multi-située permetelle aux individus de créer des liens entre des lieux aussi distants? 


\section{Les Haïtiens en France hexagonale : quel ancrage pour la plus grande communauté des Amériques?}

Cette partie décrit la manière dont les territoires de la migration haïtienne sont créés et recréés dans le contexte de la mondialisation, à l'image de la diaspora chinoise étudiée par Emmanuel Ma Mung (2012).

\section{Une croissance sensible de la population haïtienne, organisée en associations}

Dans les années 1980, cette population a connu un très fort accroissement en France ${ }^{12}$, passant de 4724 à 12311 individus (Mooney, 2008). Pour obtenir ces chiffres, la chercheuse a consulté les données de l'INSEE concernant les Haïtiens qui ne se sont pas faits naturaliser. Puis, faute de précision sur les groupes ethniques, elle a travaillé avec un statisticien de l'INSEE afin d'extraire des données détaillées. L'augmentation observée peut s'expliquer par la fuite de la dictature de Jean-Claude Duvalier (1971-1986). La décennie suivante (années 1990), l'augmentation n'a été que de 3300 individus. Quelques associations existaient déjà mais leur nombre exact n'est pas connu.

Dans les années 2000, le nombre d'associations haïtiennes et franco-haïtiennes s'établit à plus de 300 en France hexagonale pour une population estimée entre 30000 à 40 000 individus; soit une association pour 133 Haïtiens (d'après une enquête menée par l'Agence haïtienne pour le Développement local dite AHDEL) ${ }^{13}$. Le nombre de 300, indiqué également par le J.O, ne tient pas compte des associations de fait rencontrées sur le terrain. Parmi ces dernières, certaines ont été très récemment fondées, d'autres constituent des associations soit non déclarées, soit rattachées à une autre association. À titre d'exemple, le MHdF est rattaché à $M$ ap viv (du créole haïtien «Je vis ») et le Mouvement des Haïtien.ne.s de France pour le Respect de la Constitution (MOHFREC) est une association de fait.

En 2020, environ 38000 Haïtiens et 49000 Haïtiennes vivent en France, dont plus d'un tiers en Île-de-France, selon l'INSEE. Ce qui en fait la plus grande communauté issue des Amériques, avant les Brésiliens avec 61000 individus. Toutefois, en raison des entrées clandestines, nous considérons les chiffres officiels avec prudence. 
Illustration 2 : Évolution de la population haïtienne en France hexagonale entre 1980 et 2020

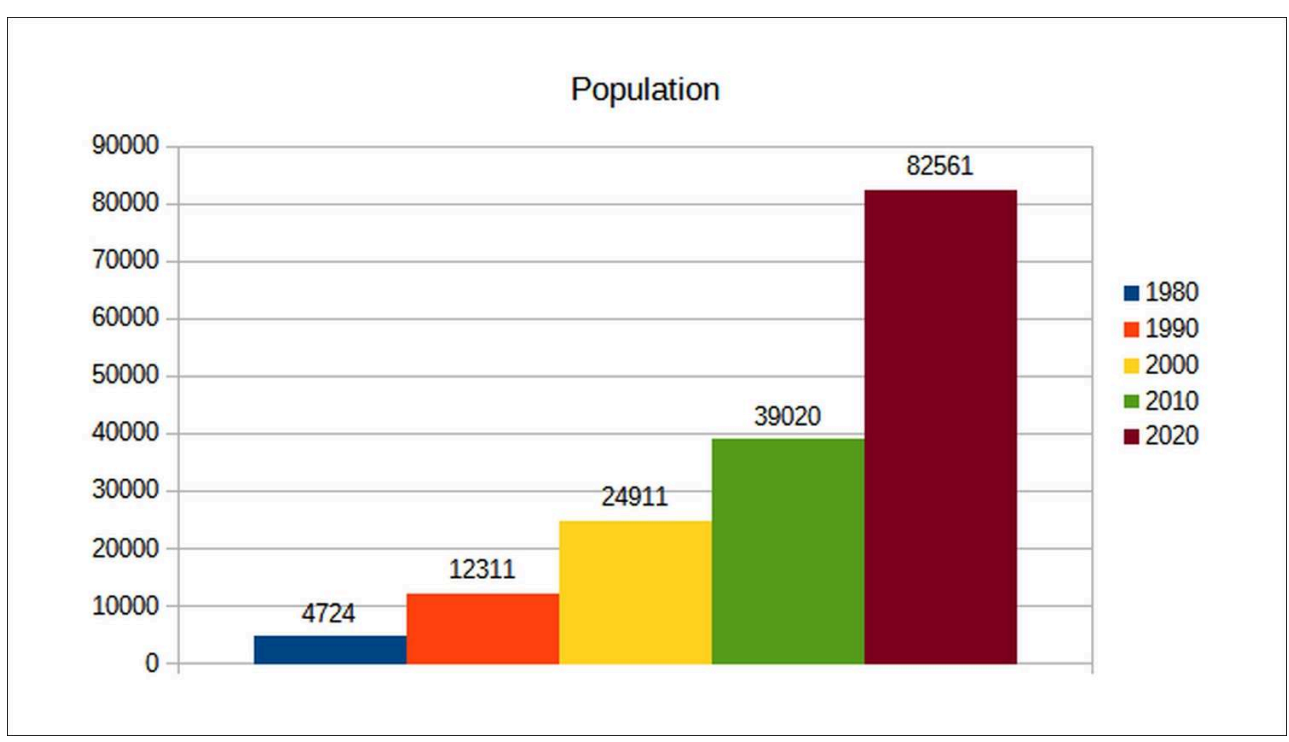

Sources : Mooney, 2008 et INSEE.

Pour suivre l'évolution de la population, nous combinons plusieurs sources représentant des périodes différentes. Nous constatons qu'au cours des périodes, la population a connu plusieurs phases d'évolution.

\section{Profil des associations haïtiennes en Île-de-France}

Ces acteurs constituent un groupe hétérogène exerçant des métiers divers parmi lesquels : enseignante, éducateur spécialisé, hôtelier. Tous sont nés et ont grandi en Haïti où ils n'avaient pas forcément d'expériences associatives. À des temporalités différentes, ils se sont engagés dans ce mouvement en France et ont créé, avec d'autres, au moins une association.

Dresser le profil d'une association revient à identifier son secteur d'activité, son domaine d'intervention et son objet. Il faut donc prendre connaissance de sa notice descriptive sur le site du J.O, consulter son statut, ou d'une manière empirique, observer les événements qu'elle organise. Après des observations portant sur vingt acteurs, nous avons relevé quatre types d'associations, présentant des similitudes avec le mouvement associatif camerounais étudié par Pierre Kamdem (2008). Dans sa typologie, l'auteur distingue les associations à caractère affinitaire, humanitaire, politique et religieux. Cette typologie semble bien correspondre à notre terrain. L'enquête de l'AHDEL menée dans les années 2000 a révélé que les secteurs d'activités des associations haïtiennes sont très variés: soutien au développement, activités confessionnelles et professionnelles, entraide et intégration (l'association Anacaona organise des permanences administratives, surtout à l'endroit des nouveaux arrivants), activités culturelles et récréatives. Mais les résultats de cette enquête ont montré qu'une association pouvait investir plusieurs secteurs d'activité.

Précisons que le caractère et la nature sont à distinguer du ou des secteurs d'activité recouverts. Par exemple, l'Association religieuse des Chrétiens à l'Extérieur d'Haïti (ARCHE), à sa création en 1984, présentait un caractère religieux, et œuvrait donc spécifiquement dans le domaine confessionnel. Devenue en 2001 l'Association pour le 
Rayonnement d'Haïti et son Environnement, elle revêt désormais un caractère humanitaire travaillant dans les secteurs culturel et de soutien au développement. Ces structures haïtiennes ont comme points communs : la rencontre de leurs membres, l'établissement de partenariat entre des associations et des individus de la communauté. Les rencontres ont, pour la plupart, lieu lors des événements politiques et socio-culturels, des commémorations et des mondanités qui jalonnent l'année civile.

Illustration 3 - Les événements marquants du calendrier de la communauté haïtienne de France

\begin{tabular}{|l|l|l|}
\hline Dates & Nature de l'événement & $\begin{array}{l}\text { Références } \\
\text { historiques }\end{array}$ \\
\hline $\mathbf{1}^{\text {"r janvier }}$ & Féte de l'Indépendance d'Haiti & $1^{\text {ct janvier } 1804}$ \\
\hline 12 janvier & $\begin{array}{l}\text { Commémoration d'un séisme de 7.3 sur l'échelle de } \\
\text { Richter }\end{array}$ & 12 janvier 2010 \\
\hline 7 avril & $\begin{array}{l}\text { Mort de Toussaint Louverture (ancien esclave devenu } \\
\text { général de l'armée indigène et précurseur de } \\
\text { l'indépendance) }\end{array}$ & 7 avril 1803 \\
\hline 18 mai & Féte de la création du drapeau haïtien & 18 mai 1803 \\
\hline 17 octobre & $\begin{array}{l}\text { Assassinat de Jean-Jacques Dessalines (Pére de } \\
\text { l'indépendance) }\end{array}$ & 17 octobre 1806 \\
\hline 18 novembre & Bataille de Vertiéres & 18 novembre 1803 \\
\hline
\end{tabular}

Auteur: R. Cadeau. territoire d'origine. Il rassemble l'ensemble des fêtes officielles et commémorations observées en Haïti et rappelle le « référent-origine » pendant toute l'année. Fabriqué à partir d'un élément fédérateur, Anthony Goreau-Ponceaud (2008) nomme "référentorigine " (là-bas), le territoire d'origine construit une continuité permettant de rapprocher les lieux de la dispersion et de les rendre contigus. En analysant la formation de l'identité transnationale chez la diaspora indienne et tamoule, l'auteur montre que l'existence d'une communauté déterritorialisée est liée à un sentiment d'appartenance. Dès lors, il explique que la diaspora prend forme grâce à « une combinaison variable de territoires » qui sont le territoire d'accueil (ici), le référentorigine (là-bas) et l'ailleurs (l'ensemble des autres lieux) et de réseaux qui s'inscrivent dans une dynamique de mouvements mais aussi d'ancrage. L'articulation des trois structures ancre les communautés diasporiques dans une relation forte avec le territoire d'origine, relation inscrite dans la longue durée.

Consciente de sa dispersion dans l'espace réel, le groupe partage une expérience collective lui permettant de mettre en scène son appartenance nationale par les commémorations, les célébrations traditionnelles et les événements observés à Paris et dans certaines communes franciliennes, aussi bien que virtuellement sur Facebook et Zoom. Lors des événements, le groupe, dont « l'élément moteur est la fabrication d'une mémoire collective» (Goreau-Ponceaud, 2008, p.4) prend corps («oekoumène diasporique » (idem)) et se forme. L'agrégation des individus symbolise la constitution du corps en dehors de sa société. On peut alors dire, comme Ma Mung (2012), que ce groupe représente un corps social dispersé qui, avant de se reproduire comme tel, se 
produit d'abord. Cette production de soi qui se joue au travers d'une représentation et d'une conscience de la dispersion, permet de former une subjectivité collective, « soit un nous identifiant » (Ma Mung, 2012) à travers l'événementiel.

31 Comparer l'animation événementielle de la communauté haïtienne avec celle d'autres communautés déjà étudiées n'est pas un exercice aisé. Les travaux s'attachent plus à présenter les projets et les actions que les événements : Christophe Daum (1998, sur la communauté malienne), Antoine Dumont (2007, sur la communauté marocaine) et Chen Ken (2016, sur la communauté chinoise). Mes recherches ne m'ont pas encore amené à comparer l'importance du calendrier des fêtes des pays d'origine des autres groupes en France afin d'évaluer leur application. Toutefois, considérant l'intensité des temps forts fonctionnant comme autant de marqueurs temporels d'une appartenance spatiale dans un contexte diasporique, on s'étonnerait que les Haïtiens soient une exception.

\section{Le corps associatif de la population haïtienne}

32 En décrivant la structuration sociale de sa communauté en France, le journaliste haïtien Wiener Kerns Fleurimond (2003, p.13) évoque des leaders politiques, des militants, des associations, des commerces, des églises, des chauffeurs de taxi, des émissions radiophoniques, des magazines, un annuaire communautaire, des restaurants, des maisons de transfert, des disquaires, des activités culturelles, littéraires, politiques, sportives.

Dans son énumération, l'auteur mêle les acteurs, les services et les lieux. Pour notre part, nous les divisons en trois ensembles dont le premier regroupe les services et les lieux, comme les maisons de transfert, les disquaires, les commerces, les espaces de célébration religieuse et les restaurants, qui sont des lieux de rencontre et de sociabilité où se retrouvent des individus, des familles, des réseaux d'interconnaissance pour entretenir la mémoire, la culture et consommer des services en relation avec le "référent-origine ». Le second ensemble réunit les acteurs, comme c'est le cas des églises et des médias qui, souvent, se constituent en association. Quant aux activités culturelles, littéraires, politiques et sportives, elles représentent des moments de sociabilité spécifiques réservés à certains profils d'individus, de familles, de réseaux ou d'acteurs comme par exemple les événements des associations étudiantes de l'Université Paris 8. Ces occasions facilitent la rencontre avec des leaders politiques, des militants, des professionnels, dont des chauffeurs de la puissante Association des Taxis haïtiens à Paris (ATHP). Toutes ces sociabilités aident à créer des liens dans le lieu d'installation dit « ici ». Voyons ce qu'il en est des liens associatifs.

\section{De nouveaux types de filiation associative, de nouvelles territorialités?}

34 Au début des années 2000, on observe une mutation des activités collectives du groupe. Avec la naissance d'une génération $\mathrm{Y}^{14}$, le tissu associatif connaît en effet de nouvelles configurations traduites par l'apparition d'une dizaine de structures (selon les calculs de l'auteure), dont cinq sont présentées dans la figure 3. Elles ont comme particularité une filiation numérique, une mise en réseau des acteurs, une volonté d'être plus visibles dans les espaces réel et virtuel que les associations traditionnelles. Ce qui va de 
pair avec l'utilisation intense des TICs (Jolivet, 2017) type internet, web, média en ligne, applications dédiées, Facebook, Whatsapp, Zoom, etc. Un tel usage des TICs semble contribuer à reconfigurer ce "tout petit monde» dans la mesure où les petites et moyennes associations peuvent se regrouper avec des individus pour former d'autres structures de taille plus importante, surtout dans l'espace virtuel. Les échanges s'intensifient et les informations se diffusent plus rapidement et plus largement. La logique de la mondialisation accélère le temps collectif au sens où elle facilite des débats en réunion dans un espace-temps qui n'a pas besoin d'être formalisé.

Au-delà de la question du temps chronologique ou collectif, les TICs donneraient également plus de visibilité en permettant aux associations traditionnelles de par leur filiation (réunion et démarchage des adhérents dans l'espace réel) de mobiliser et de fédérer davantage de militants. Aussi se caractérisent-elles par une concentration d'acteurs, en regroupant plusieurs associations sous une nouvelle dénomination et en recrutant divers individus pour la formation d'une plus grande structure. L'exemple du CORECOHF, qui compte plus de 50 adhérents, illustre parfaitement ce phénomène (illustration 4). Par ailleurs, le nombre d'adhérents par association est très variable. Il y va de deux ou trois (pour une petite association, souvent isolée) jusqu'à 50 pour une " grande » association capable d'en fédérer d'autres. Selon la loi du $1^{\mathrm{er}}$ juillet 1901, deux signatures suffisent pour déclarer une association. D'ailleurs le formulaire à remplir par les chargés de l'administration mentionne trois fonctions principales (président, secrétaire, trésorier).

Dans la dynamique actuelle, on remarque de nouveaux types de filiation qui sont tantôt numérique, tantôt associatif. Non seulement des anciennes associations en génèrent de nouvelles, mais des forums de débats en engendrent aussi. Les procédés de création à l'œuvre observés sont de trois ordres représentant trois configurations : création de nouvelles associations ; fédération de plusieurs associations; formation d'un groupe associatif (qui se rapproche du modèle des groupes médias, il réunit des associations dirigées par le même président ou comité administratif). Souvent, les nouvelles structures adoptent une nouvelle dénomination, sont de taille plus importante et agissent à des échelles différentes (illustration 4). Par exemple, les associations Centre de Recherche et d'Innovation sociale (CRIS), M'ap viv, Les Pierres semées et d'autres forment le MHdF ; le MCU est créé par les responsables de CRIS et d'autres associations, mais regroupe des Haïtiens résidant au Canada, aux Etats-Unis, au Mexique, aux Antilles françaises. À leur tour, ils invitent d'autres compatriotes à les rejoindre ou à participer, entre autres, aux primaires organisées le 27 décembre 2020 en prélude aux élections présidentielles en Haïti.

37 Par ailleurs, on assiste également au rattachement de nouvelles associations à des anciennes. Une tactique qui peut être vue comme l'absorption d'une structure par une autre. Néanmoins, ce scénario avantage certains responsables en leur permettant de rattacher le "nouveau-né » par une décision collective en assemblée générale et les fondateurs en leur évitant les démarches administratives pour la déclaration. Car, par cette procédure, la nouvelle structure devient une association déclarée même si elle n'a pas déposé de statuts à la préfecture. Elle trouve son existence légale à travers celle qui l'absorbe. Le MHdF rattaché à $M$ ap viv en est un exemple.

Une autre nouvelle configuration est la naissance de fédérations d'associations. C'est le cas de la Plate-forme des Associations franco-haïtiennes (PAFHA) créée en 2002 avec dix associations. Aujourd'hui elle en compte $200^{15}$. Son objectif est de faciliter la mise en 
réseau des associations œuvrant pour Haïti depuis la France. Dès sa création, elle devait être un espace où les structures gardent leur identité et leurs activités propres mais aussi partagent leurs expériences, s'enrichissent de celles des autres et recherchent des solutions aux difficultés entravant le développement d'Haïti.

La dernière configuration est celle où les acteurs créent un groupe associatif dans lequel chaque entité revêt un caractère propre dans un ou plusieurs secteurs distincts. Par exemple, MCU et Espace-HVIE, qui sont de la même génération et de la même lignée (illustration 4), présentent, un caractère politique (MCU) et un caractère humanitaire (Espace-HVIE). Le MCU investit le secteur de soutien au développement et l'EspaceHVIE se positionne dans les secteurs d'entraide et d'intégration, dans les activités culturelles et récréatives.

Illustration 4 - Généalogie de structures haïtiennes créées en île-de-France (2018-2020)

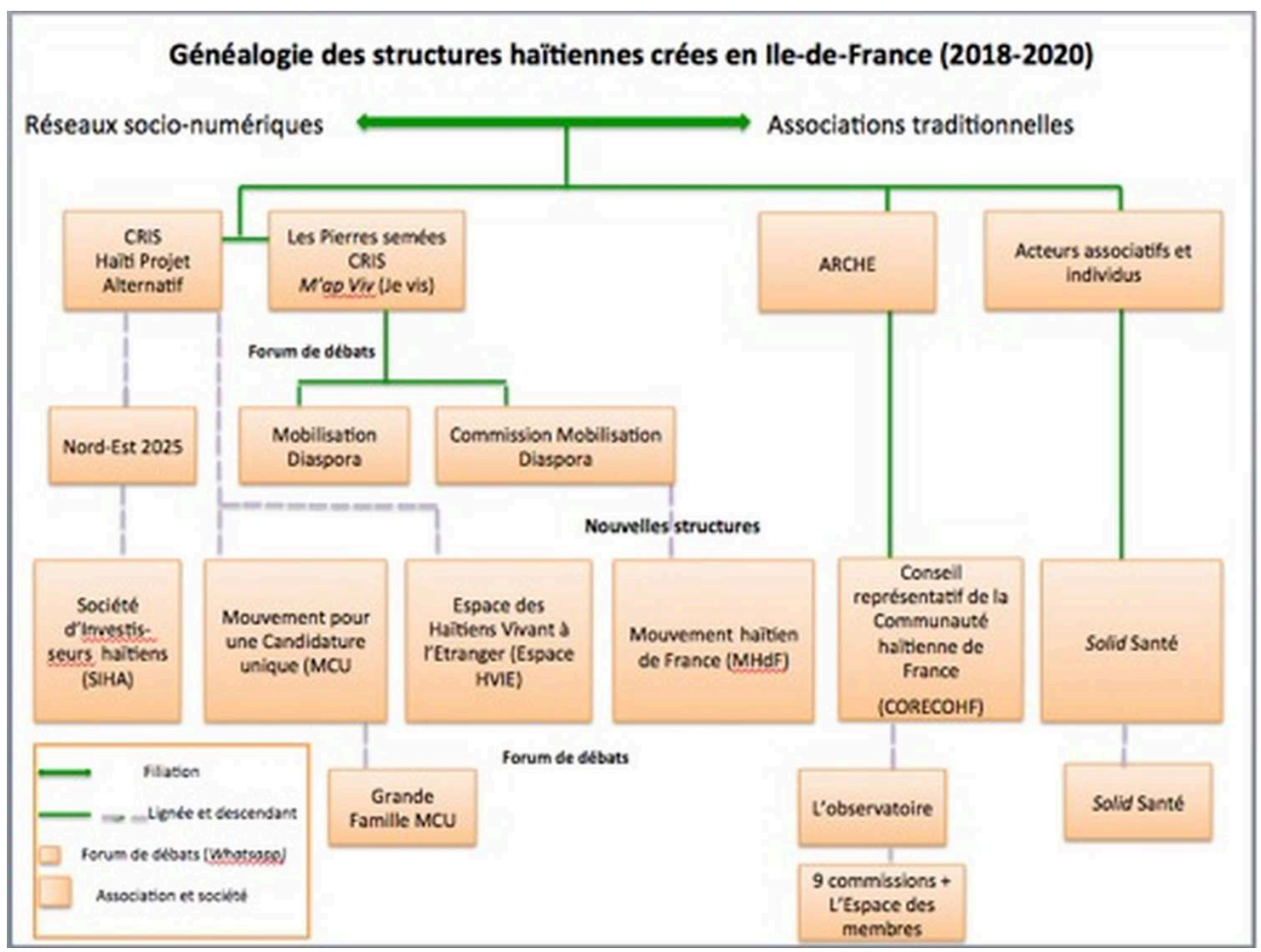

Source : enquête de terrain 2019-2020. Auteur : R. Cadeau.

Cette généalogie permet de visualiser les types de filiation des deux générations d'associations. Deux proviennent de la rencontre des individus dans l'espace réel (CORECOHF et Solid ${ }^{16}$ Santé) tandis que trois (MCU, Espace-HVIE, MHdF) émanent d'un espace virtuel (l'application Whatsapp). L'application est utilisée par les acteurs pour générer des forums de débats et pour créer des associations, parfois sous fond de tensions et de rivalités. MCU, Espace-HVIE, MHdF sont des exemples de nouvelles associations reconnues par la loi 1901.

Dans une telle dynamique, le monde numérique apparait comme un cyberespace de la relation entre des individus éloignés de leur pays d'origine et permet ainsi «des échanges directs et instantanés pour les acteurs » (Hassane, Berthomière, 2009, p. 229). Sur les forums Whatsapp, les individus échangent surtout sur l'actualité et sur la situation politique d'Haïti en partageant des vidéos, des photos, des messages vocaux, 
des articles, notamment issus des journaux haïtiens accessibles en ligne comme Le Nouvelliste et Rezonodwès (Réseau Nord-Ouest en français) à des temporalités variables. Par exemple, sur HPA, les débats se tiennent quotidiennement tandis que des sujets hebdomadaires sont proposés sur Nord-Est 2025 et Grande Famille MCU. Ce qui peut vouloir dire que les deux derniers sont moins visités.

Les participants réagissent surtout par des messages vocaux, rarement en français : le créole haïtien reste la langue dominante. Sur la préférence de l'oral à l'écrit, nous formulons l'hypothèse qu'elle s'explique par la fonction émotive et la «facilité » de l'oral, par le fait que le créole est commun aux adhérents et par la possibilité qu'offrent les applications d'interagir rapidement. Quant à la gestion du temps et de la parole, les animateurs de HPA, Nord-Est-2025 et Grande Famille MCU exigent que leurs adhérents respectent le sujet du jour et que les messages ne dépassent pas 1'30 mn. Sur ces trois forums, la diffusion de contenu et d'expression autre que par les messages vocaux est formellement interdite. Sur d'autres, tels que L'Observatoire du CORECOHF et HPJ, les débats sont lancés à des temporalités aléatoires en fonction de l'actualité politique, des projets et sur libre initiative des adhérents. Cette mise en relation entre «ici », « làbas » et « ailleurs » est-elle propre à la communauté haïtienne ?

Dans une perspective comparative, nous souhaitons savoir si d'autres associations de migrants présentent la même configuration spatiale. Nos recherches nous ont conduit sur le site du Forum des Organisations internationales issues des Migrations (FORIM), dont la PAFHA fait partie, qui est dirigé par l'ancien président de cette dernière. Créé en 2002, le FORIM fédère environ 700 associations intervenant au MAGHREB, en Afrique subsaharienne, en Asie du Sud-Est, aux Caraïbes et dans l'Océan indien ${ }^{17}$. Il constitue un bel exemple de regroupement ou de fédération d'associations ayant des pratiques transnationales inscrivant leurs actions « ici » et «là-bas ». Il montre alors un double ancrage à la fois international, et local, puisque son siège social et la plupart de ses adhérents se trouvent en Île-de-France. De par ses relations en France, le FORIM jouit d'une reconnaissance et d'une visibilité à différentes échelles : à l'échelle locale, les acteurs publics et les associations locales; aux échelles départementale et régionale, les Conseils du Val-de-Marne et d'Île-de-France ; à l'échelle internationale, l'Organisation internationale des Migrations. Composé de migrants d'origines géographiques diverses et pouvant ne pas être tous émaner de "peuple diasporisé », il nous paraît prudent de n'établir de similarité entre la diaspora haïtienne et les groupes du FORIM que sur les plans des pratiques transnationales et d'organisation permettant une "co-présence " et un "ancrage multiple». En ce qui a trait aux différences, outre la taille des structures, la mise en scène de l'identité reste un élément majeur.

Cédric Audebert (2011) en montrant que l'espace migratoire haïtien révèle un polycentrisme et une réticularité, avance que la métropolisation de l'espace haïtien est l'un des éléments ayant donné accès aux «supports technologiques modernes de circulation et de communication, dans des grandes villes bien intégrées aux réseaux du système-monde (...) ». Considéré comme une compétence, l'accès aux technologies s'avère également un savoir d'usage permettant de faire société à l'ère de la mondialisation. Aussi, l'appropriation de l'espace numérique par les réseaux d'acteurs semble participer à la structuration spatiale par-delà les frontières, au même titre que l'église et l'entreprenariat. Dès lors, les associations apparaissent comme un moyen de rapprochement entre les Haïtiens et leurs territoires, comme l'a démontré, d'une part, Violaine Jolivet (2017) dans ses analyses de l'usage des TICs par la communauté 
haïtienne à Montréal. D'autre part, Souley Hassane et William Berthomière (2009) soutiennent que grâce aux TICs le migrant connecté sent une proximité en dépit de la distance : la « co-présence », c'est-à-dire le « sentiment d' " être là », essaie de prendre le dessus afin de défaire « le sentiment de la double absence de l'immigré (...) analysée par [le sociologue] Abdelmalek Sayad.» (p. 229). Dans le prolongement des réflexions dépassant l'image du migrant " déraciné », Dana Diminescu (2002) établit une analogie entre les «comportements migratoires contemporains" et le "modèle d'électron libre » utilisant les TICs en l'occurrence le téléphone portable afin de se déplacer « sans se détacher de son atome social » ou « vivre ensemble à distance » (Proulx, 2008).

Parallèlement aux forums, les émissions audio-visuelles représentent également des espaces de paroles privilégiés comme Kòn Lanbi ${ }^{18}$ sur Fréquence Paris Plurielle ou Transparans (traduit par transparence) sur $H$ Live rendant quotidien le "référent origine ». La mise en relation via les médias traditionnels et en ligne semble induire, dans le groupe haïtien, une reconfiguration territoriale due, non seulement, à une filiation numérique des associations mais aussi à un " ancrage multiple » des individus (Jolivet, 2017), engendrant de nouvelles territorialités. Dès lors, l'étude des liens renvoie à une analyse de l'imbrication d'« ici », de "là-bas » et d' "ailleurs". Si l'articulation du local et du global privilégie l'analyse du processus soulignant des capacités à "faire société » dans la dispersion (Hassane, Berthomière, 2009), qu'est-ce que les pratiques spatiales de la génération $Y$ de la communauté étudiée en offrent comme illustration?

Illustration 5 - Structures haïtiennes et leurs relations spatiales (2018-2020)

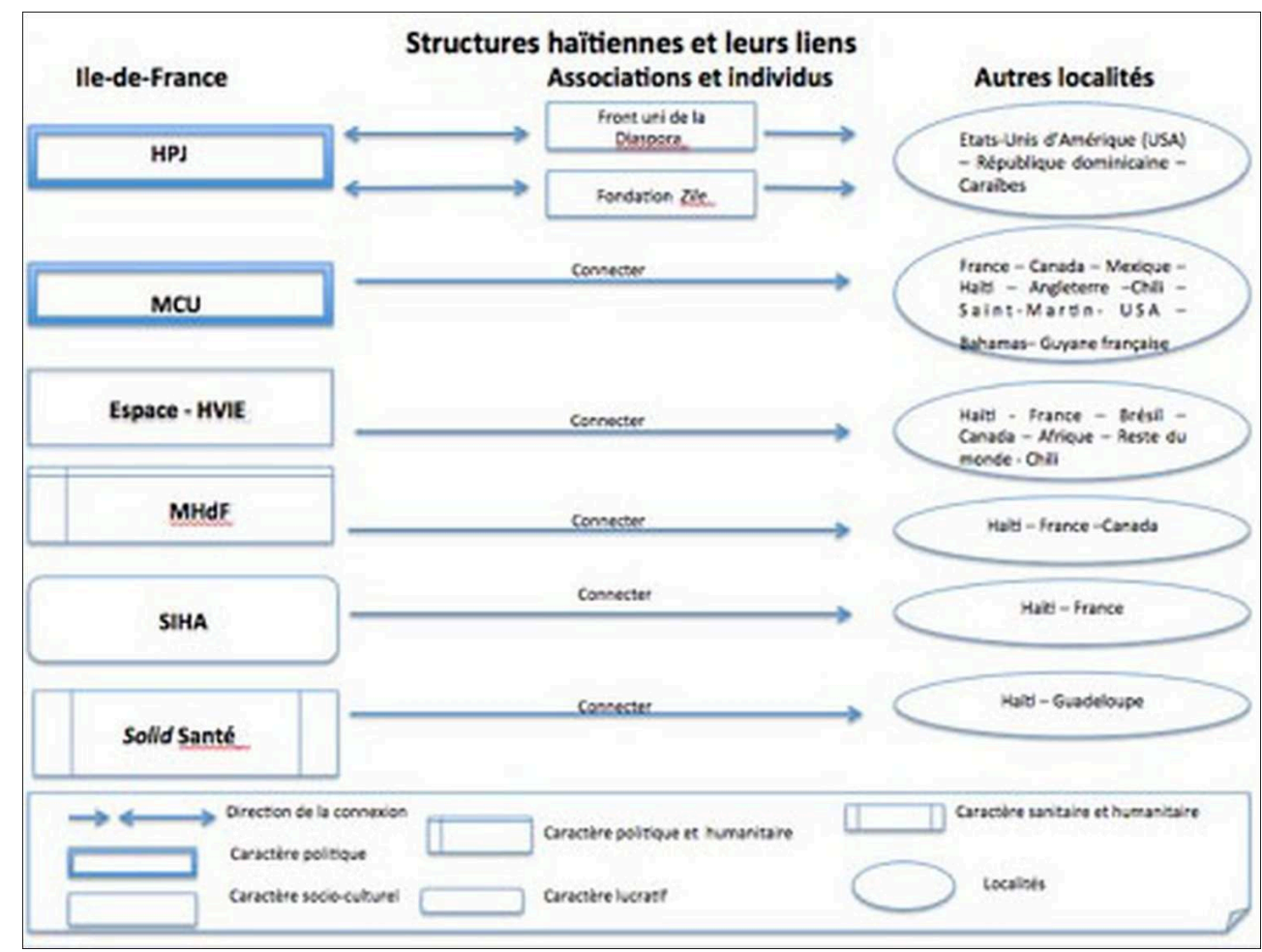

Source : enquête de terrain 2019-2020. Auteur : R. Cadeau.

L'illustration 5 montre la connexité des lieux d'installation de la migration haïtienne. D'un côté, les associations prennent l'initiative de la mise en relation des lieux même si, 
souvent, la connexion est établie avec des individus, qu'ils soient adhérents ou sympathisants. Ils participent aux débats, échangent des informations concernant l'association et le pays d'origine, contribuent aux frais des projets et parfois votent aux élections. La connexion établie avec les autres pôles sert souvent à l'organisation de projets transnationaux en France, en Haïti ou ailleurs, comme le congrès du Front uni de la Diaspora haïtienne à Washington. Notons par ailleurs que les nouveaux pôles, comme le Chili ou le Mexique figurent dans cette nouvelle configuration.

Emmanuel Ma Mung (2012) met en évidence des relations « interpolaires » migratoires, économiques, informationnelles ou affectives au sein de la diaspora chinoise témoignant de la connaissance des individus sur d'autres lieux et d'autres semblables installés en dehors de la terre d'origine. L'auteur considère cette connaissance comme étant déjà une "représentation de la dispersion par ces individus » au même titre que "les histoires familiales, les différents média, les productions culturelles, littéraires ou cinématographiques et les travaux portant sur la diaspora (...) », qui est l'expression du même type de connaissance. Il ajoute que la presse communautaire, les associations et les communautés virtuelles participent aussi dans la représentation de la dispersion. Néanmoins, il reconnaît que « la constitution d'un cyberespace diasporique » participe « à la création d'un imaginaire transnational » capable d'entraîner " un nationalisme déterritorialisé et un chauvinisme ethnique » dans certains cas.

La question de la représentation de la dispersion d'une diaspora à l'échelle de l'individu semble se poser en de mêmes termes à l'échelle de groupe. Elle peut aussi bien être démontrée par des associations dans la performance d'un "ancrage à distance » renvoyant à «des lieux et maisons significatives » qui ne sont pas présents (Ramos, 2006) mais restent accessibles grâce à des pratiques transnationales.

\section{Quelles pratiques transnationales, à quelles échelles?}

À travers leurs événements, les associations mettent en œuvre une diversité de pratiques culturelles, sociales et politiques transnationales. Elles prennent tantôt la forme de partenariat, tantôt celle de regroupement informel, permettant aux acteurs d'agir à différentes échelles spatiales. Par exemple, en avril 2020, Espace-HVIE et MHdF ont organisé une collecte de fonds auprès de la communauté pour approvisionner la prison des femmes à Cabaret, au nord-ouest de Port-au-Prince. La différenciation entre ces pratiques s'établit à partir des types d'événements (socio-culturel, religieux, politique, humanitaire, colloque et conférence, etc.), et des formes (rassemblement, messe, soirée, conférence, colloque, défilé carnavalesque, récital, concert, bal, etc.) que revêtent les activités en général. Aussi peuvent-elles être transcrites dans les discours des acteurs lors des événements ou des prises de parole sur les réseaux socionumériques. Les discours aident à décliner la façon dont les individus en migration articulent leur présence ainsi que leurs actions à la fois à l'échelle locale (en France) et à l'échelle internationale (Haïti et dans les autres lieux de la migration). Cette articulation est mise en évidence par les pages Facebook de l'ARCHE et de HPJ, montrant une façon de se rendre présents dans plusieurs espaces géographiques, en même temps, malgré la distance. Nous avons sélectionné quelques données résultant de notre ethnographie numérique, en mêlant textes et images, afin montrer ce qui est donné à voir dans cet espace. 
Illustration 6 - Montage des pages d'accueil des associations ARCHE et HPJ

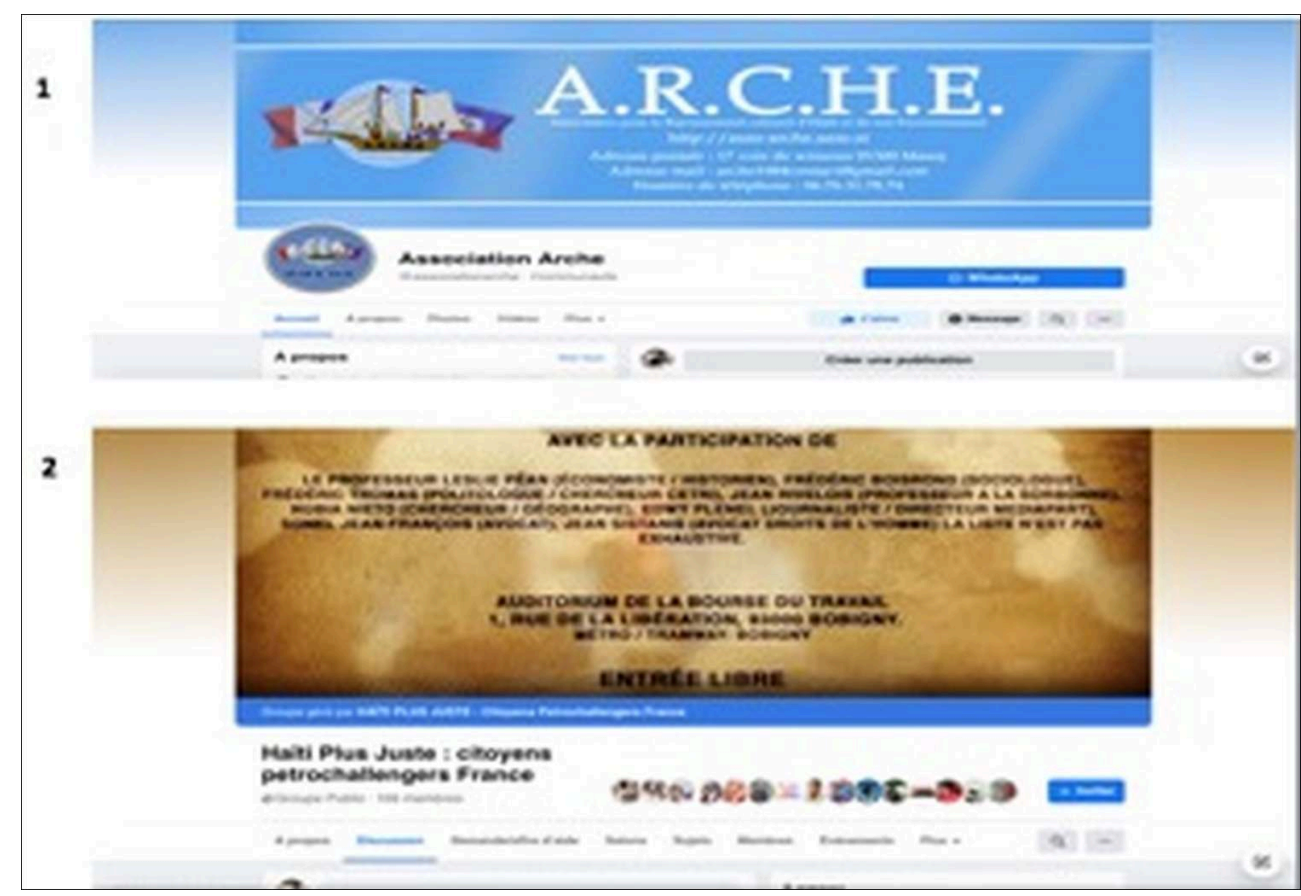

Sources : captures d'écran des pages d'accueil sur Facebook.URL : https://www.facebook.com/ associationarche; https://www.facebook.com/groups/337926366865274.

50 L'illustration 6 présente les images de la page de l'ARCHE et celle du groupe HPJ sur Facebook. La première illustre l'ancrage de l'association sur Facebook ainsi que sur Whatsapp et la deuxième uniquement sur Facebook. La capture du haut informe sur la définition de l'acronyme de l'ARCHE, sa localisation géographique, son numéro de contact et ses coordonnées et références électroniques. La capture du bas met en vue le plus grand événement réalisé par HPJ en affichant les personnalités du comité scientifique et le lieu de l'événement. Les militants de HPJ s'identifient au mouvement Petro Challenge (réclamant en 2019 au président Jovenel Moïse des explications sur l'utilisation qu'il a fait des 3,8 milliards de dollars prêtés par le président Hugo Chavez à Haïti) ${ }^{19}$ en ajoutant citoyens petrochallengers France (les militants de Petro Challenge) au nom du groupe. L'association détient une page et un groupe sur Facebook sur lesquels on observe majoritairement des publications en lien avec Haïti: des articles, des affiches, des images et des vidéos produits depuis Haïti ou d'autres lieux de la migration. Le partage et la diffusion des contenus permettent de remarquer une mise en relation des lieux d'établissement des Haïtiens ; une connexion entre l'île-de-France Haïti et ailleurs.

\section{De l'île-de-France à Haïti : faire pays}

\section{Participer à la vie politique}

On observe assez classiquement que les sièges sociaux des associations ne correspondent pas aux lieux où se déroulent les événements. D’ailleurs, un « responsable associatif $»^{20}$ a déclaré que nombre d'acteurs préfèrent se domicilier à Paris afin de bénéficier d'une meilleure visibilité et d'une meilleure image. La localisation du siège peut donc avoir une valeur symbolique en lien avec la 
représentation du lieu. Dans le corpus présenté, CRIS, Espace-HVIE, MCU et CORECOHF sont domiciliés à Paris tandis que leurs présidents résident en banlieue. Ces associations travaillent tantôt pour la promotion d'Haïti en France, ou du moins en Îlede-France, tantôt pour agir directement et concrètement en Haïti. Celles issues de la vague de 1957-1986, sous la dictature, avaient un fort caractère politique et donc pour objectif de provoquer des changements en Haïti (Mooney, 2011). Plus récemment, dans le contexte de pandémie Covid-19, Espace-HVIE, aussi localisé à Port-au-Prince, a organisé une distribution de masques dans ses locaux haïtiens au mois de mai 2020. Il s'agit bien d'agir localement en s'appuyant sur des ressources et des réseaux transnationaux.

Sur le terrain, les acteurs en lien direct avec la politique contribuent à marquer leur territoire en Haïti, comme pour HPJ. Le président de HPJ explique, sur Facebook, son entretien avec un journaliste de Port-au-Prince en mission de reportage à Paris sur la crise politique haïtienne :

[La] Radio Télévision Caraïbes FM est venue jusqu'à Paris pour connaître la position de la communauté Haïtienne en France, le journaliste m'a rencontré en tant qu'acteur engagé et président de l'association Haïti Plus juste accompagné de Willer Pierre Rivière membre de HPJ. Nous lui avons exprimé nos inquiétudes face à la situation si triste en Haïti. Nous avons réitéré notre position d'accompagner le peuple haïtien qui exige une vie meilleure. Nous lui informons de notre lutte contre la corruption et les dérives sociales en Haïti. Par contre, la violence meurtrière en Haïti nous est inacceptable, (...). Voilà pourquoi, nous avons prôné un dialogue inter Haïtien pour redéfinir l'autre modèle de gouvernance [écrit par Samuel Colin, le 19 novembre 2019].

53 Fondée en août 2018, juste après le mouvement social contre la hausse du prix du carburant en Haïti, HPJ a tenu des rassemblements et des manifestations politiques à Paris pour prolonger les mobilisations. À la Bourse du Travail de Bobigny, elle a également organisé, une conférence internationale en juin 2019 sur la corruption en Haïti. Elle a été dirigée par l'ancien président de la Fédération de la Diaspora haïtienne d'Europe (FEDHE), lequel a également co-fondé le Front uni de la Diaspora haïtienne aux États-Unis (à Washington en 2013). Le post de l'association affirme sa position dans la crise pendant qu'il sous-tend sa reconnaissance, ou du moins celle de son président. Le post confère à son auteur un positionnement d'acteur sur l'échiquier politique national depuis l'étranger. La prise de position publique dans la vie politique du pays d'origine indique la présence et l'ancrage de l'association et de ses adhérents sur le territoire haïtien.

54 Nos observations révèlent la velléité, chez nombre d'acteurs associatifs, de participer activement à la gestion politique et à l'organisation de la vie sociale du pays d'origine. Le post offre une illustration de cette velléité politique articulant, dans un premier temps, une "co-présence» (Diminescu, 2002) en France et en Haïti ; puis, dans un second temps, une «présence démultipliée » dans les autres «dimensions de l'espace haïtien » (Anglade, 1995) voire dans les espaces virtuels en construisant un cyberespace haïtien.

Toutefois, faire de la politique à l'extérieur du pays ne représente pas les mêmes enjeux que sur place, dans la mesure où les risques, les émotions et les représailles ne sont évidemment pas les mêmes. La volonté politique des Haïtiens de l'étranger s'apparente à l'affirmation d'une « haïtianité » dans le cas où elle est exprimée dans les autres pôles de cette migration comme aux États-Unis où un ensemble de marqueurs spatiaux ont 
signifié la présence du groupe (la création du journal Haïti Progrès en 1983). Sans nier la réalité d'une sorte d'unité, produite par les effets identitaires, articulant une solidarité réelle entre les individus d'« ici » et de "là-bas ", Goreau-Ponceaud (2008) reconnaît que ce sentiment d'identité commune, dans un contexte diasporique, peut faire l'objet d'une logique d'instrumentalisation par l'État (les acteurs institutionnels tels le Ministère des Haïtiens vivant à l'étranger (MHAVE), les autorités consulaires et diplomatiques). D'un autre côté, une forme d'instrumentalisation "par le bas " peut également émaner des pratiques associatives elles-mêmes sans que cela soit exclusif.

\section{Donner une autre image d'Haïti}

Malgré une indéniable aura des intellectuels, écrivains et artistes haïtiens en France, la pauvreté reste, depuis les années 1980, l'entrée de « toute présentation (médiatique et autres) sur ce pays » (Mérat, 2019). Mais cette image, souvent relativisée par les acteurs associatifs, est, d'une part, compensée par des actions de promotion de la culture et de l'art (Revue Pour Haïti) et de vulgarisation historique (le site internet de l'association Haïti Mémoire et Culture) entreprises par certaines associations et médias communautaires. D'autre part, des acteurs visent à proposer un contre-discours lors des événements ou sur les réseaux socionumériques, afin de visibiliser leurs actions de développement et de solidarité à l'égard du pays d'origine. Dès lors, ils dévoilent aussi leur implication politique « là-bas ».

«C'est ce que nous faisons à l'ARCHE, [c'est] pour rendre au paysan haïtien, à

l'habitant, sa dignité et son humanité. C'est pour cela que nous réfléchissons à la pérennité de nos actions. Nous pensons que le Conseil représentatif de la Communauté haïtienne de France peut garantir cette pérennité. Organisons-nous ", [post publié sur Facebook, le $1^{\text {er }}$ décembre 2019 par ARCHE].

Ce post met en exergue un rapprochement entre «là-bas », représenté par « le paysan haïtien », et l'île-de-France, « ici ». En effet, le siège social de l'ARCHE se situe dans le département de l'Essonne, à Massy, ce qui le localise dans une ambiance de lointaine banlieue sud de Paris. L'association s'attache à rendre présente la ruralité haïtienne à l'aide d'expositions de photos et d'affiches exhibant la dimension "paysanne ». Cela dévoile l'imaginaire de la ruralité et celui de la paysannerie haïtienne, estimée à $60 \%$ de la population en 2016, selon la Banque de la République d'Haïti. Le secteur "agriculture, sylviculture et pêche" représente environ $20,35 \%$ du PIB ${ }^{21}$. Lors des événements caritatifs, l'ARCHE donne à voir des pratiques et savoir-faire quasiment made in Haïti (danses, musiques, peintures, plats traditionnels). Les rentrées d'argent, déclare le président, permettent de réaliser des projets divers comme la construction d'infrastructures scolaires (Ecole Agapao à Martissant, au sud de Port-au-Prince) ou artistiques (Parvis des arts et des savoirs à Colline Deslandes, à l'est de la commune de Léogâne).

\section{De l'île-de-France au reste du monde : une imbrication des échelles spatiales au service de «l'haïtianité »}

En analysant l'« externalisation " des réseaux sociaux des communautés haïtiennes vivant à l'étranger caractérisée par un système spatial d'échanges, Audebert (2011) écrit qu'ils concordent à "une nécessité de survivre " qui permet de prendre «son destin et celui de sa famille et de sa localité » en dehors du pays, en même temps qu'on 
tient à conserver la structuration du «lien social originel» (p.3). Dès lors, l'organisation collective des émigrés, de sorte à assurer une continuité dans l'espace et dans le temps, trouve une explication dans une redéfinition du rapport du peuple haïtien à son territoire et à la migration. L'illustration 7 témoigne d'une imbrication des échelles spatiales par les acteurs associatifs dans leur volonté d'agir en articulant le local, le national, le continental et l'international.

Illustration 7 - Capture d'écran de la page d'accueil du site internet de Espace-HVIE

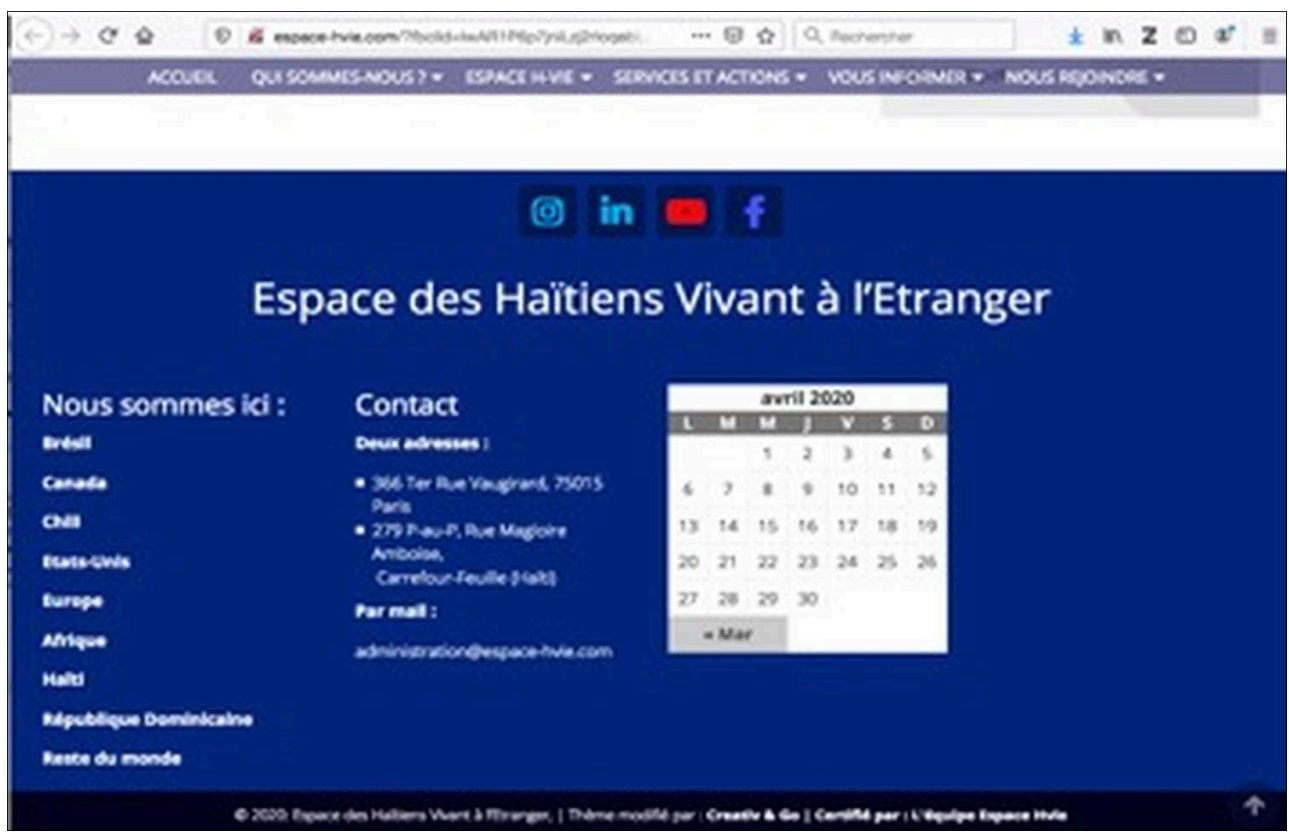

URL?

Cette page illustre plusieurs éléments. D'abord, la dénomination de l'association qui ressemble à celle du MHAVE, traduit, d'une certaine façon, une volonté de fédérer les Haïtiens autour d'une mémoire s'apparentant à l'incarnation symbolique de la mère patrie. L'Espace-HVIE apparaît ainsi comme un portail par lequel tout Haitien peut contacter des compatriotes. C'est aussi une forme de prise de conscience de l'extension territoriale de son peuple, puisque les lieux d'établissement des communautés haïtiennes, y compris les nouveaux pôles (Brésil, Chili), sont nommés. Quant aux adresses, elles renvoient à une logique de double ancrage dans la dimension physicospatiale de l'espace migratoire. En dernier lieu, la représentation offre une illustration de l'externalisation des réseaux et des liens des adhérents ainsi que des militants connectés sur whatsapp.

\section{Conclusion}

Le but de cet article, rappelons-le, est de montrer que l'articulation entre la dimension physico-spatiale et la dimension numérique de l'espace permettent de redéfinir les territorialités via les TICs dans un contexte migratoire. Elle invite à penser la diaspora haïtienne au prisme d'une telle articulation en mobilisant les analyses faites sur d'autres groupes connectés en situation diasporique ou de "diasporisation ». Comme nous l'avons vu, après 2010, de nouveaux pôles ont émergé dans les espaces migratoires 
haïtiens, surtout en Amérique du Sud, dessinant une nouvelle géographie. Au-delà du séisme, ce phénomène peut s'expliquer par l'accroissement des problèmes d'infrastructures, d'insécurité mais aussi par l'épidémie de choléra liée à la présence des Casques bleus et ayant infecté plus d'un demi-million d'Haïtiens la même année : autant de facteurs qui ont contraint des milliers d'habitants à quitter le pays. L'association Espace-HVIE, étant localisée sur des lieux «réels " et "virtuels», se présente donc comme l'un des points de ralliement dans la mesure où elle est représentée comme un élément fédérateur des Haïtiens d'« ici » et de «là-bas » avec ceux de l'« ailleurs ».

61 À l'inverse, une migration de travail importante a attiré des milliers d'ONG et de professionnels étrangers en Haïti (Klarreich, Polman, 2012). S'intéressant aux mobilités des élites du Nord dans les pays du Sud comme Haïti et Timor-Leste, particulièrement les travailleurs de l'humanitaire, Marie Redon (2010) relate qu'il y "existe un type spécifique de mobilités inhérentes au secteur du développement, de l'aide humanitaire et à l'omniprésence des organisations intergouvernementales et non gouvernementales » (p. 209). Malgré les différences spatiales entre les deux pays en termes d'espace et de temps (situation géographie et histoire coloniale), la géographe insiste sur des caractéristiques communes à savoir « un positionnement national et international de second ordre » qui fait du Timor-Leste un territoire marginalisé, mais aussi connecté au monde notamment par les travailleurs internationaux, comme Haïti.

Mais la connexion d'Haïti présente une autre forme fabriquée par une diversité

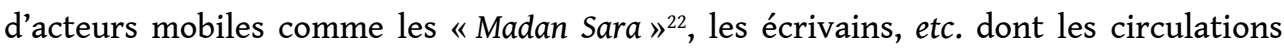
schématisent en creux des sens et des directions différents. Ils peuvent aussi bien aller du Sud au Nord que du Sud au Sud et leur mouvement migratoire est susceptible de dessiner : soit un déplacement d'un point A vers un point B; ou un déplacement d'un point $A$ vers un point $B$ en passant par d'autres points; ou encore un aller-retour entre deux points au minimum. En ce sens, Nathalie Bernardie Tahir et Sarah Bernard (2020) avancent que plusieurs recherches sur les mobilités démontrent «la porosité des catégories de mobilité » mais aussi un " continuum mobilitaire » allant de migrations courtes à d'autres plus ou moins longues. La nouvelle dynamique associative de la communauté haïtienne en France dévoile quelques-uns des «artisans » de cette forme de connexion d'Haïti au reste du monde. En outre, elle traduit un ancrage territorial avec néanmoins une présence démultipliée des acteurs à une échelle transnationale. Les discours de ces derniers illustrent une présence à la fois physique et symbolique tant dans l'espace public constitué que dans les lieux interconnectés. Les groupes, s'inscrivant également dans une auto-affirmation en tant qu'acteur transnational, via les réseaux socionumériques, donnent à voir en même temps leur capacité d'action audelà des frontières. Il convient de noter également que les connexions entre les associations « ici », "là-bas » et "ailleurs " révèlent implicitement les interactions sociales (entre les acteurs) et spatiales (entre les lieux où se trouvent les acteurs) de la migration internationale haïtienne.

Sur la question des territorialités, Georges Anglade énonce, lors d'un entretien ${ }^{23}$, qu'en cartographiant la distribution de la musique haïtienne sur le continent américain, on peut découvrir une forme de conurbation territoriale. L'axe part de Port-au-Prince, traverse la mer des Caraïbes pour remonter la côte-est états-unienne jusqu'à Montréal. Dans le prolongement de ce raisonnement, nous constatons qu'au regard de la territorialisation de la communauté haïtienne en Île-de-France, une conurbation 
virtuelle distribuant les associations dans le cyberespace de cette diaspora est bel et bien émergente.

\section{BIBLIOGRAPHIE}

Anderson B. (Dauzat P. E., trad.), 2006. L'imaginaire national. Paris, La Découverte, 224 p.

Anglade G., 1974. L'espace haïtien. Montréal, Presses universitaires du Québec, 222 p.

Anglade G., 1982a. Espace et liberté en Haïti. Montréal, ERCE et Centre de Recherches Caraïbes, p. 144 .

Anglade G., 1982c. Atlas critique D'Haïti. Montréal, ERCE et Centre de Recherches Caraïbes, Université de Montréal, $80 \mathrm{p}$.

Anglade G., 1990. Carte sur table. Vol. 1. Itinéraires et raccourcis. Cinq ans de jalons : 1977-1981. Port-auPrince, New York, Montréal, Éditions Henri Deschamps, Études et recherches critiques d'espace, Éditions ERCE et Georges Anglade, 200 p.

Anglade G., 1995. L'espace haïtien. Mon pays d'Haïti. Port-au-Prince, Les Éditions des Alizés, 56 p.

Audebert C., Calmont A., 2003. Logiques, réseaux, enjeux des migrations internationales dans le Bassin caraibe. Mappe-Monde 72 [En ligne], p. 25-28. https://www.researchgate.net/publication/ 26431702_Logiques_reseaux_enjeux_des_migrations_internationales_dans_le_Bassin_caraibe

Audebert C., Calmont A., 2007. Immigration antillaise et diversité sociale de la population noire aux États-Unis. In : Calmont A., Audebert C. Dynamiques migratoires de la Caraibe. Paris, Géode Karthala, pp.77-92. URL: https://halshs.archives-ouvertes.fr/halshs-00817538/document Audebert C., 2007. L'immigration caribéenne aux Antilles françaises : des modes d'insertion différenciés. In Calmont A., Audebert C. Dynamiques migratoires de la Caraibe. Paris, Géode Karthala, p 169-179.

Audebert C., 2011. La diaspora haïtienne : vers l'émergence d'un territoire de la dispersion ?. In Célius C. A., Le défi haïtien : économie, dynamique sociopolitique et migration. Paris, L'Harmattan, p. 193-212.

Audebert C., 2012. La diaspora haïtienne. Territoires migratoires et réseaux transnationaux. Rennes, Presses universitaires de Rennes, $195 \mathrm{p}$.

Bastide R., Morin F., Raveau F. et al, 1974. Les Haïtiens en France. Paris, La Haye, 229 p.

Béchacq D., 2007. La diaspora haïtienne à Paris : significations, visibilités et appartenances. In Calmont A., Audebert C. Dynamiques migratoires de la Caraibe. Paris, Géode - Karthala, p. 253-271.

Béchacq D., 2010. Pratiques migratoires entre Haïti et la France. Des élites d'hier aux diasporas d'aujourd'hui. EHESS, thèse de doctorat en anthropologie.

Bidegain G., 2013. Les Haïtiens qui sont partis. Combien sont-ils ? Où sont-ils ? [En ligne : Université UQAC, Les Classiques des sciences sociales]. URL: http://classiques.uqac.ca/ contemporains/Bidegain_Gabriel/Haitiens_qui_sont_partis/Haitiens_qui_sont_partis_texte.html 
Calmont A., 1993. Les Haïtiens en Guyane : une communauté en voie d'intégration ?. Espace, populations, sociétés, $\mathrm{n}^{\circ}$ 2, p. 427-434. URL: https://www.persee.fr/doc/ espos_0755-7809_1993_num_11_2_1604 - DOI: https://doi.org/10.3406/espos.1993.1604

Calmont A., 2007. Trajets socio-identitaires chez les jeunes issus de la migration haïtienne en Guyane. Cuadernos Interculturales, vol. 5, n 9, p. 9-27.

Calmont R., Gorgeon C., 1987. L’immigration haïtienne en Guyane. Equinoxe, n² 23, p 1-16.

Casséus Eybalin C. R., 2013. Les migrants, acteurs transnationaux du développement : les associations haïtiennes en France et jamaïcaines au Royaume-Uni. Université de Poitiers, thèse de doctorat en géographie.

Cossée C., 2010. Médias tsiganes en France et en Hongrie : re-présentation de soi dans l'espace public. Revue européenne des Migrations Internationales, vol. 26, n 1, p. 57-79.

Dagnaud M., 2013. Génération Y. Les jeunes et les réseaux sociaux, de la dérision à la subversion. Paris, Les presses de Sciences Po, $210 \mathrm{p}$.

Daum C., 1998. Les associations de Maliens en France. Migration, développement et citoyenneté. Paris, Karthala, 253.

Delachet-Guillon C., 1996. La communauté haïtienne en Île-de-France. Paris, L'Harmattan, 192 p.

Dejean P., 1990. D’Haïti au Québec. Montréal, CIDIHCA, 204 p.

Diminescu D., 2002. L'usage du téléphone portable par les migrants en situation précaire. Hommes et Migrations, $\mathrm{n}^{\circ} 1240$, p. 66-79 p.

Domenach H., 1985. Les migrations intra-caribéennes. Revue européenne des migrations internationales, vol. 2, $\mathrm{n}^{\circ}$ 2, p. 9-24. URL: https://www.persee.fr/doc/ remi_0765-0752_1986_num_2_2_1096 - DOI: https://doi.org/10.3406/remi.1986.1096

Dumont A., 2007. La marocanité associative en France. Militantisme et territorialité d'une appartenance exprimée à distance. Université de Poitiers, thèse de géographie.

Exavier R., 2020. Mobilité sociale et politisation de la diaspora haïtienne en France et au Québec. Université Paris Sorbonne, thèse de doctorat.

Fleurimond W. K., 2003. La communauté haïtienne de France. Dix ans d'histoire : 1991-2001. Paris, L'Harmattan, 327.

Girault M., Dubost I., Calmont A., et al., 2009. La Guadeloupe et la Martinique dans l'histoire française des migrations en régions de 1848 à nos jours. Hommes \& migrations [En ligne]. URL: http://journals.openedition.org/hommesmigrations/252 - DOI: https://doi.org/10.4000/ hommesmigrations.252

Gorgeon C., 1985. Immigration clandestine et bidonvilles en Guyane, les Haïtiens à Cayenne. Revue européenne des migrations internationales, vol. 1, $\mathrm{n}^{\circ}$ 1, p. 143-158. URL: https://www.persee.fr/doc/ remi_0765-0752_1985_num_1_1_970 - DOI: https://doi.org/10.3406/remi.1985.970

Gouëset V., Hoffmann O., 2006. « Communauté. Un concept qui semble poser problème dans la géographie française ». In Séchet R., Veschambre V. Penser et faire la géographie sociale. Rennes, Presse universitaire de Rennes, p. 263-275.

Goreau-Ponceaud A., 2008. La diaspora tamoule : lieux et territoires en Île-de-France. L'Espace Politique [En ligne], vol. 4, n 1, p. 19-33. URL: https://journals.openedition.org/espacepolitique/ 495 - DOI: https://doi.org/10.4000/espacepolitique.495 
Haddad M., 2018. Migration DOM-métropole des années 1960 à nos jours : itinéraires d'une minorité française. Institut d'Etudes politiques, thèse de doctorat en sociologie.

Hartog T., Desse M., 2009. Les littoraux de la Caraibe. Pratiques sociales et nouvelles dynamiques spatiales. Paris, Karthala, Géode, 228 p.

Hassane S., Bertomière W., 2009. Les cyber-diasporas à l'heure de la mondialisation. In Jaffrelot C. et al., L'enjeu mondial, Presses de Sciences Po, p. 225-234.

Hubon L., 1987. Comprendre Haïti. Essai sur L'État, la nation, la culture. Port-au-Prince, Henri Deschamps-Karthala, $174 \mathrm{p}$.

Icart J. C, 1987. Négriers d'eux-mêmes. Essai sur les boat people haïtiens en Floride. Montréal, Editions du CIDIHCA, $188 \mathrm{p}$.

Icart J. C., 2017. La migration haïtienne dans le monde. Chemins critiques, vol. 6, nº 1, p. 57-74.

Jackson O. R., 2011. Geographies of the Haitian Diaspora. New York, Routledge, 348 p.

Jadotte H., 1977. Haitian Immigration to Quebec. Journal of Black Studies, vol. 7, n 4, p. 485-500.

Jean R., 2006. Atlas des populations immigrées en Guyane, Paris, ACSE.

Jolivet V., 2015. Miami la cubaine. Géographie d'une ville-carrefour entre les Amériques. Rennes, Presses universitaires de Rennes, $271 \mathrm{p}$.

Jolivet V., 2017. Médias et migration. Territorialités connectées et ancrages au sein de la communauté haïtienne de Montréal (1960-2016). Espace populations sociétés [En ligne]. URL: http://journals.openedition.org/eps/7202 - DOI: https://doi.org/10.4000/eps.7202

Kamdem P., 2008. Le mouvement associatif de la diaspora camérounaise. Enjeux et perspectives. Paris, L'Harmattan, $223 \mathrm{p}$.

Klarreich K., Polman L. La République ONG d'Haïti. Le novelliste [En ligne]. URL: https:// lenouvelliste.com/article/110873/la-republique-ong-dhaiti

Ken C., 2016. Les associations chinoises. Hommes \& migrations [En ligne]. URL: http://

journals.openedition.org/hommesmigrations/3629 - DOI: https://doi.org/10.4000/

hommesmigrations.3629

Laëthier M., 2011. Être migrant et Haïtien en Guyane. Paris, CTHS, 320 p.

Lamare J., 2019. Le projet migratoire des immigrants haïtiens de Saint-Denis : le prix d'une humanité exilée. Université Paris Sorbonne, thèse de doctorat en sciences sociales et philosophie de la connaissance.

Lanno-Cyr S., 2016. Regards sur les territoires haïtiano-montréalais : les impacts des pratiques transnationales au quotidien. Université de Montréal, mémoire de maîtrise en géographie.

Louidor E. W., 2011. L'Amérique du sud : pôle d'attraction de la migration haïtienne. Vivre ensemble. Le Webzine [En ligne]. URL: https://cjf.qc.ca/vivre-ensemble/webzine/article/ lamerique-du-sud-pole-dattraction-de-la-migration-haitienne/

Louis-Jacques. R., 2015. Les Haïtiens de France : état des lieux et perspectives. Haïti Monde, vol. 9, $\mathrm{n}^{\circ}$ 23-24, p. 6-9.

Lucien G. E., 2009. Espaces périphériques et économie d'archipel : la trajectoire contemporaine de Verrettes (Haïti). Port-au-Prince, Montréal, Editions de l'UEH et du CIDHICA.

Lucien G. E., 2015. Little Haiti, si loin de Dieu et si près du centre-ville de Miami. Port-au-Prince, Imprimeur S.A, 236 p. 
Lucien G. E., 2018. Le Nord-Est d'Haïti. La perle d'un monde fini : entre illusions et réalités (Open for Business). Paris, L'Harmattan, 347 p.

Madiou T., 1989. Histoire D’Haïti. Port-au-Prince, Henri Deschamps, Tome II (1799-1803), 558 p.

Ma Mung E., 1994.Non-lieu et utopie : la diaspora chinoise et le territoire. Espace Geographique, vol. 23, n 2, p. 106-113. URL: https://www.persee.fr/doc/spgeo_0046-2497_1994_num_23_2_3280

Ma Mung E., 2012. Continuité temporelle, contiguïté spatiale et création d'un monde propre. Le cas de la diaspora chinoise. L'Espace géographique, vol. 1, p. 352-368. URL: https://www.cairn.info/ revue-espace-geographique-2012-4-page-352.htm

Marchandise S., 2010. La construction diasporique marocaine sur le web. Enjeux méthodologiques d'une nouvelle approche des migrations. Migrations Société, vol. 6, $\mathrm{n}^{\circ} 132$, p. 125-138. URL: https://www.cairn.info/revue-migrations-societe-2010-6-page-125.htm - DOI: https://doi.org/10.3917/migra.132.0125

Mérat P. J., 2020, Etre pauvre en Haïti. Les Cahiers d'Outre-Mer [En ligne], nº 279, p. 27-49. URL : http://journals.openedition.org/com/9806 - DOI : https://doi.org/10.4000/com.9806

Milia-Marie-Luce M., 2007. La grande migration des Antillais en France ou les années BUMIDOM. In Calmont A., Audebert C., Dynamiques migratoires de la Caraibe. Paris, Karthala, Géode Caraïbe, p. 93-103.

Mooney M., 2008. Structures de médiation et intégration des immigrants haïtiens à Paris. Revue européenne des migrations internationales, vol. $24, \mathrm{n}^{\circ} 1$, p. 89-114.

Piantoni F., 2016. Trente ans d'immigration en Guyane. Un processus d'intégration sociale et économique sous contrainte. Après-demain, vol. 3, n 39, p. 27-31. URL: URL : https:// www.cairn.info/revue-apres-demain-2016-3-page-27.htm - DOI: https://doi.org/10.3917/apdem. 039.0027

Proulx S., 2008. Des nomades connectés : vivre ensemble à distance. Hermès La Revue, vol. 2, $\mathrm{n}^{\circ} 51$, p. 155-160. URL : https://www.cairn.info/revue-hermes-la-revue-2008-2-page-155.htm

Ramos E., 2006. L'invention des origines. Sociologie de l'ancrage identitaire. Paris, Armand Colin, 221 p.

Redon M., 2010. Mobilis in mobili : des vies « en mobilité » au Sud. Espace populations sociétés [En ligne], $\mathrm{n}^{\circ}$ 2-3, p. 209-220. URL: http://journals.openedition.org/eps/4098 - DOI: https://doi.org/ $10.4000 /$ eps.4098

Rokotoary S., 2019. Usages de Facebook dans l'événementialisation de la construction d'une communauté transnationale. Communiquer [En ligne], $\mathrm{n}^{\circ}$ 25, p. 23- 37. URL: http:// journals.openedition.org/communiquer/4000 - DOI: https://doi.org/10.4000/communiquer.4000 Romanovski Z., Piantoni F. Les stratégies d'accès au logement des Haïtiens dans l'agglomération de Cayenne comme facteurs de restructuration urbaine. L'Espace Politique [En ligne], $\mathrm{n}^{\circ}$ 6. URL: http://journals.openedition.org/espacepolitique/1009 - DOI https://doi.org/10.4000/ espacepolitique.1009

Romanovski Z., 2016. Les réseaux de migrants haitiano-guyanais dans l'espace américain. Paris, L'Harmattan, $324 \mathrm{p}$.

Saint Victor A., 2018. De l'exil à la communauté : une histoire de l'immigration haïtienne à Montréal (1960-1990). Université du Québec à Montréal, mémoire de maîtrise en histoire.

Smorag P., 2019, Les espaces haïtiens de Miami : les défis d'une appropriation territoriale en terre américaine. Les cahiers d'Outre-Mer [En ligne], n²79. URL: http://journals.openedition.org/com/ 10331 - DOI : https://doi.org/10.4000/com.10331 
Tahir N. B., Bernard S., 2020, Faire voile et jeter l'ancre en Polynésie française. Carnets de géographes [En ligne], n 14. URL: http://journals.openedition.org/cdg/6037 - DOI: https:// doi.org/10.4000/cdg.6037

Trouillot L., 2010. Chronique. Haïti, vivre avec la mort. Le Point [En ligne], 19/01/20210. URL: https://www.lepoint.fr/culture/haiti-vivre-avec-la-mort-par-lyonel-

trouillot-19-01-2010-415015_3.php\#

Trouillot L., 2019. Tribune. Silence complice sur Haïti : solitude des morts sans importance.

L'Humanité, 2.10.2019. URL: https://www.humanite.fr/lyonel-trouillot-silence-complice-sur-haitisolitude-des-morts-sans-importance-678016.

Trouillot L., 2020. Entretien. Le peuple a besoin du soutien international. L'Humanité, 13/01/2020. URL: https://www.humanite.fr/lyonel-trouillot-le-peuple-besoin-du-soutien-

international-683023

Trouillot L., 2021. Tribune. Haïti : Appel à solidarité pour peuple en danger. L'Humanité, 8/02/21. URL: https://www.humanite.fr/haiti-appel-solidarite-pour-peuple-en-danger-699818

Trouillot L., 2021. Entretien. Lyonel Trouillot, écrivain : tout citoyen haitien est en danger. Télérama. URL : https://www.telerama.fr/debats-reportages/lyonel-trouillot-ecrivain-toutcitoyen-haitien-est-en-danger-6819282.php

\section{NOTES}

1. 1 Du créole haïtien, se traduit littéralement par «crâne rasé ». Dans un registre familier, l'expression peut vouloir dire aussi «allègrement », « indéfiniment ». En nommant leur parti ainsi, les fondateurs du PHTK, en particulier l'ancien président-chanteur, Michel Martelly fait à la fois référence à sa coiffure et à la pérennité de ce nouveau groupe politique.

2. Voir dans la bibliographie un corpus d'articles de Lyonel Trouillot, publiés dans la presse française, décrivant la crise politique en Haïti et faisant office d'appel à l'aide.

3. L'utilisation de la communauté ici correspond au sens courant des sciences sociales qui, dans la tradition de l'École de Chicago, considèrent l'identité collective, l'appartenance à un groupe social ou l'attachement à des cultures

spécifiques comme des fondements du lien social caractérisant une communauté. En outre, Vincent Gouëset et Odile Hoffmann (2006) soutiennent que « désignant un "groupe de personnes", la communauté n'a pas

d'échelle a priori. Elle peut avoir une pertinence au niveau local le village, le quartier, régional ou international - les diasporas par exemple» (p. 263). Vu que notre étude porte sur des associations haïtiennes distribuées dans plusieurs départements de la région francilienne, nous employons le terme communauté au regard des marqueurs d'une affirmation identitaire, de l'existence de liens sociaux culturels et de l'entretien d'une mémoire collective.

4. Selon une recherche avec pour mot-clé « association haïtienne » sur le site du Journal officiel de la République française (J.O.). URL: https://www.journal-officiel.gouv.fr/associations/ recherche/resultats/?

5. Le site de l'Organisation de Coopération et de Développement économique (OCDE), www.oecdilibrary.org.

6. Il est à préciser que la phase de l'enquête dont nous parlons ici ne concerne pas les associations religieuses haïtiennes. Cette enquête a été menée auprès des associations non religieuses, c'est-à-dire qui ne professent pas et qui n'exercent pas de pratiques religieuses lors de leur assemblée 
7. L'expression "socionumérique » est utilisée pour souligner que «les aspects sociaux et numériques sont deux dimensions intrinsèquement constitutives du phénomène observé [c'est-àdire la territorialisation des acteurs associatifs haïtiens en Ile-de-France] » (Sarah Rokotoary, 2019, p. 30).

8. La crise de 1929 et la Grande Dépression ont entrainé une baisse d'activité ralentissant les flux migratoires et la livre de sucre « est passée de 5,50 dollars à 22,50 centimes de dollar US ", Lucien, 2015 : p. 72. Aussi les migrants ont été expulsés. Environ 30000 Haïtiens sont rentrés au bercail de 1936 à 1937, après un massacre d'au moins 20000 compatriotes en République dominicaine (Anglade, 1982).

9. Un terme inventé pour l'occasion, selon Icart (2017).

10. De 1963, date du premier voyage des Haïtiens vers ce territoire (Calmont, 1993) à 1999, la population guyanaise compte, parmi les immigrants, $28 \%$ de ressortissants de ce groupe (Jean, 2006).

11. Voir Sa Ellison, 2019, Chèche lavi, film, 1h16mns. Du créole haïtien, chèche lavi traduit par " chercher la vie », se débrouiller et induit l'idée de mobilité. Le film retrace la trajectoire de deux jeunes ayant quitté Haïti pour atteindre les États-Unis en passant par le Brésil et le Mexique.

12. Nous considérons qu'il s'agit de la France hexagonale. Parce que non seulement la chercheure a travaillé sur l'agglomération parisienne mais les chiffres ne concordent pas avec ceux d'autres recherches, notamment celles sur la Guyane ou les statistiques de l'INSEE sur la Guyane. Par exemple, l'INSEE indique que la part des Haïtiens représentait $29 \%$ du total des immigrés de la Guyane en 1982, soit 5452 sur 18803.

13. Le site de l'AHDEL, URL : http://haitiensenfrance.free.fr/spip.php?rubrique25

14. Cette appellation désigne les individus nés entre 1980 et 1990 " au moment où l'usage de l'internet s'est généralisé » (Dagnaud, 2013). On considère que « l'e-culture est leur royaume et, pour cette raison, on les désigne par le terme digital natives » (Dagnaud, 2013., p. 7).

15. Le site de la Plateforme des Associations franco-haïtiennes, URL: http://www.pafha.fr

16. Du créole haïtien, se traduit par solide en français.

17. Le site de la Coordination sud, URL : https://www.coordinationsud.org

18. Nom donné à la coquille du mollusque marin lobatus gigas servant d'instrument de musique et rassemblement en temps de lutte et de révolte. Le Nègre marron (marron inconnu), sculpture située en face du Palais national à Port-au-Prince, tient une machette et souffle dans un kòn lanbi.

19. Le site de RFI, URL: https://www.rfi.fr/fr/ameriques/20180820-fonds-petrocaribe-haitiensdemandent-comptes-classe-politique

20. J'utilise ce terme, familier aux militants associatifs, dans le sens de personnes actives et influentes ayant des responsabilités et des rôles comme Maud Laëthier (2011) l'a fait dans le cas des associations haïtiennes en Guyane et je souligne que ces individus ressemblent des meneurs poussant les autres membres de leurs associations à agir, souvent dans leur sens.

21. Le site de l'Organisation des Nations-unies pour l'alimentation et l'agriculture, URL: http:// www.fao.org/haiti/fao-en-haiti/le-pays-en-un-coup-doeil/fr/

22. Traduit littéralement par " Madame Sarah », vient du nom local donné à un oiseau et désigne les commerçantes informelles haïtiennes circulant entre la campagne et l'agglomération de Portau-Prince ou entre l'international et le pays (Hartog, Desse, 2009).

23. Sur le site de "Ile en Ile », URL: http://ile-en-ile.org/georges-anglade-5-questions-pour-ileen-ile/ 


\section{RÉSUMÉS}

Cet article cherche à comprendre les impacts socio-spatiaux des usages des Technologies d'Informations et de Communication (TIC) dans un contexte diasporique. L'étude des usages des applications dédiées et des réseaux socionumériques, par des associations, permet d'analyser les façons dont les Haïtiens se rendent présents « ici ", « là-bas » et « ailleurs " afin de continuer à «faire pays» dans les «territoires de la dispersion». Nous explorons les mécanismes de structuration du groupe autour d'un « référent origine " grâce auquel les migrants « font corps » en dehors de leur société en y restant attachés et connectés à distance.

This article seeks to understand the socio-spatial impacts of Information and Communication Technologies (ICT) utilization in a diasporic context. The study of the uses of dedicated applications and socio-digital networks by diaspora associations, allows us to analyze the ways in which Haitians make themselves present "here ", "there» and "elsewhere ", in order to continue " making their country » in the "territories of dispersal». We explore the mechanisms of structuring the group around an "origin referent" through which migrants become a « social formation » outside their society, while remaining attached and connected to it remotely.

\section{INDEX}

Keywords : Haitian migration, diaspora, territoriality, île-de-France, Association, ICT Mots-clés : migration haïtienne, diaspora, territorialité, île-de-France, association, TIC Thèmes : Sur le Champ

\section{AUTEUR \\ RÉBECCA CADEAU}

Rébecca Cadeau, rebeccalecadeau@gmail.com, est doctorante et chargée d'enseignement à l'Université Sorbonne Paris Nord, UFR LLSHS, Laboratoire Pléiade. 